http://doi.org/10.11646/zootaxa.4158.3.2

http://zoobank.org/urn:Isid:zoobank.org:pub:3FA278A1-1407-47BE-9A79-DEFF9A03B3E4

\title{
The Neotropical tanyderid Araucoderus gloriosus (Alexander) (Diptera, Tanyderidae), with description of the egg, larva and pupa, redescription of adults, and notes on natural history
}

\author{
R. ISAÍ MADRIZ \& GREGORY W. COURTNEY \\ Department of Entomology, Iowa State University, Ames, IA 50011-3222, USA
}

\begin{abstract}
Larvae, pupae and adults of Araucoderus gloriosus (Alexander) were collected during fieldwork in Chilean Patagonia, December 2013 and January 2014. Eggs were obtained from females that oviposited in captivity. Association of all life stages is based on co-occurrence and rearing of individual larvae to adults. A diagnosis for the genus and species is provided. Descriptions of the egg, larva and pupa and redescriptions of the male and female are completed. Eggs of $A$. gloriosus are the first described for Tanyderidae. Natural history characteristics for this species, including microhabitat, copulatory behavior and oviposition, are discussed.
\end{abstract}

Key words: aquatic, morphology, immature, primitive crane flies, Chile, Patagonia

\section{Introduction}

Tanyderidae Osten Sacken 1880 is comprised of 38 extant species divided among 10 genera, with representatives in the Australasian (21 species), Oriental (three species), Afrotropical (one species), Palearctic (six species), Nearctic (four species) and Neotropical (three species) regions.

Since discovery of the first extant tanyderid (Osten Sacken 1859), information pertaining to the biology or behavior of this family has been of great interest, but fragmentary. Except for studies by Exner \& Craig (1976) and Lukashevich \& Scherbakov (2014), most published information about tanyderid natural history includes brief mention of habitat or life history (Colless \& McAlpine 1970; Krzemiński \& Judd 1997; Judd 2004) usually in the context of a species description (Alexander 1930; Crampton 1930; Wood 1952; Knight 1963; Rose 1963; Podeniene \& Gelhaus 2013;) or geographic range extension (Alexander 1935).

Information about the reproductive biology of primitive crane flies, particularly regarding mating behavior and oviposition, has been hypothetical at best. Knowledge of natural history is lacking partly due to sampling difficulties for adults and especially immature stages. Larvae of some species are known to burrow in soft, saturated wood (Hinton 1966; Colless \& McAlpine 1970, 1991; Krzemiński \& Judd 1997; Lukasevich \& Scherbakov 2014) while others are free living in the shallow hyporheic zone of cobble-, gravel- and sand-bottom streams (Alexander 1930; Crampton 1930; Wood 1952; Knight 1963; Rose 1963; Exner \& Craig 1976; Anthon 1988; Krzemiński \& Judd 1997; Judd 2004; Courtney \& Merritt 2008; Marshall 2012). A single larva of Protanyderus stackelbergi Savchenko (1971) was recorded from a leaf pack (Podeniene \& Gelhaus 2013), but it is unclear if this is the primary larval habitat for the species. Pupae have been found on the damp ridges above the water line of sandy banks of streams and rivers (Wood 1952; Knight 1964). Adult tanyderids are rarely collected, cryptic and believed to be short lived, which contributes further to a poor understanding of the family.

Previous descriptions of tanyderid larvae and pupae have been fragmentary (Alexander 1930; Crampton 1930; Rose 1963; Knight 1963, 1964; Exner \& Craig 1976; Anthon 1988; Wipfler et al. 2012; Podeniene \& Gelhaus 2013; Lukashevich \& Shchervankov 2014, 2016), with only three definitive larva-adult associations: Protoplasa fitchii (Alexander 1930; Crampton 1930), Peringueyomyina barnardi (Wood 1952) and Tanyderus pictus (Lukashevich \& Shcherbakov 2016; Madriz unpublished observation). Eutanyderus wilsoni larvae (Colless \& 
McAlpine 1970, 1991) and pupae (Hinton 1966) have been identified, but no detailed description has been published. Descriptions of adult tanyderids have been based mainly on wing venation and coloration (Alexander 1927), with terminalia descriptions being superficial or absent.

Araucoderus Alexander, 1929 is one of three monotypic tanyderid genera in the Neotropical region: Neoderus Alexander, 1928 and Tanyderus Philippi, 1865. Of these, Araucoderus gloriosus (Alexander 1920) was originally placed in Tanyderus, and later Alexander (1927) transferred the species to Radinoderus Handlirsch, 1909. Subsequently, Alexander (1929) proposed Araucoderus as a genus to include A. gloriosus. Despite the species being reclassified multiple times, the immature stages remain undescribed and, aside from wing venation, little is known about adult morphology. Moreover, no information has been recorded regarding the natural history of this species.

During a recent expedition to southern Chile, we collected specimens of all life stages of A. gloriosus and obtained eggs from ovipositing captive females. Detailed observations were made of larval, pupal and adult habitat and behavior. In the current paper, we redescribe A. gloriosus adults, describe its eggs, larvae, and pupae, and provide details of the behavior, habitat and development of the species. This is the first study including complete descriptions of the morphology and natural history for all life stages of any tanyderid species.

\section{Material and methods}

Study area. This project focused Chilean Patagonia where samples were taken from streams and their riparian vegetation. Localities range from montane to lowland between latitudes $39^{\circ} 31^{\prime} \mathrm{N}$ and $48^{\circ} 02^{\prime} \mathrm{N}$, including Araucanía (Region IX), Los Ríos (Region XIV), Los Lagos (Region X) and Aysén (Region XI) (Fig. 2).

Material. All life stages of Araucoderus were examined. Most specimens were collected between December, 2013 and January, 2014. Additional larval specimens were collected during October, 2007. Adult specimens were borrowed from the National Museum of Natural History, Smithsonian Institution, Washington, D.C. (USNM). Voucher collections from the current study are deposited in the following: USNM; Academy of Natural Sciences, Philadelphia, PA (ANSP); Iowa State Insect Collection, Ames, IA (ISIC); Canadian National Collection of Insects, Ottawa (CNCI) and Museo Nacional de Historia Natural, Santiago, Chile (MNNC).

Adult specimens were hand-collected or swept from riparian vegetation. Pupae were collected by hand from the wet root mats of marginal vegetation and damp marginal substrata, while larvae were collected in kick samples and by hand-picking them from marginal substrata. Definitive associations of all life stages are based on rearing of individual larvae to adult emergence. Species associations of non-reared adult males and females were based on observed copulation and morphological similarity of wings. Association of eggs was based on two copulations and resulting eggs from two different females.

Specimen preparation. Field-collected and reared adults were fixed primarily in $70 \%$ or $95 \% \mathrm{EtOH}$, whereas most larvae and pupae were killed either in $95 \% \mathrm{EtOH}$ or by immersion in hot water and subsequent placement in $95 \% \mathrm{EtOH}$. Eggs were preserved in $70 \% \mathrm{EtOH}$ at different times after oviposition to address color changes. Undissected adult specimens and remaining dissected structures were dehydrated in a graded ethanol series (95, $100,100,100 \%$ ), followed by a graded hexamethyldisilazane (HMDS) series (25, 50, 100, 100\%). Morphological studies were based on partial- or whole-animal preparations, slide mounts, and scanning electron microscope (SEM) micrographs of selected body parts. For selected specimens, the head and terminalia were macerated in a solution of cold potassium hydroxide (approximately 10\%) and slide mounted permanently or temporarily using Canada balsam or glycerin, respectively.

Light microscopy. Specimens were observed using an Olympus SZX-9 and SZX-12 stereo microscopes and a Nikon Eclipse E800 compound microscope, the latter equipped with differential interference contrast and both fitted with an ocular micrometer. Drawings were rendered with the aid of a drawing tube on both systems. Photomicrographs were captured using a Nikon DS-Fil mounted interchangeably on both microscopes. Composite images were generated using NIS-Elements 3.2 imaging software and edited in Adobe Photoshop ${ }^{\circledR}$ 12.1x64. Illustrations and plates were produced using Adobe Illustrator ${ }^{\circledR}$ CS5 15.1.0 and Adobe Photoshop ${ }^{\circledR}$ 12.1x64.

Scanning electron microscopy. The head capsule, thorax and genitalia were dissected from select adult specimens while the head capsule and anal division were dissected from select larval specimens. All material was sonicated in EtOH for 5-10 seconds. After sonication, specimens were dried either chemically with HMDS or by 
using a critical point drier, then sputter coated with gold-palladium. Material was examined using a JEOL 5800LV Scanning Electron Microscope.

Terminology. Larval terminology follows Courtney et al. (2000) and Wipfler et al. (2012), with head sensilla numbered from anterior to posterior and dorsal to ventral. Pupal terminology follows Borkent (2012). Descriptions of adult morphology follow Cumming \& Wood (2009). Terminology of the male genital tract follows Borkent \& Sinclair (2012). The parameres in Tanyderidae are complex, with multiple elements herein referred to as "dorsal bridge of paramere", "dorsomedial element of paramere", "parameral lobe at gonocoxite base" and "lateromedial element of paramere". Female post abdomen terminology follows Cumming \& Wood (2009) and (Kotrba 2000). In the material examined section, "instar IV larvae" and "pupae" are abbreviated below as "L" and "P", respectively. We use the term "natural history" in reference to all observational data.

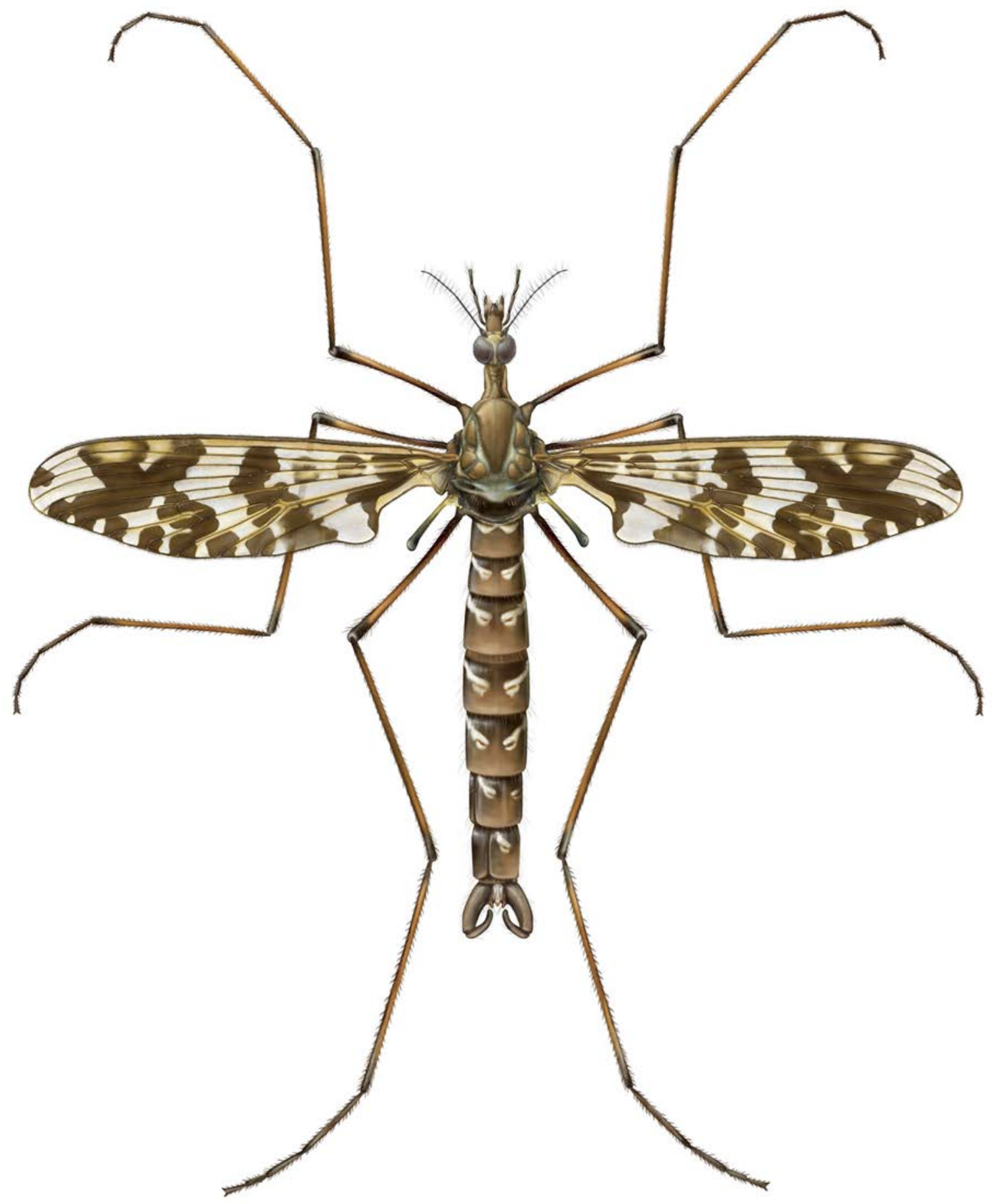

FIGURE 1. Araucoderus gloriosus (Alexander) male habitus, dorsal view. 


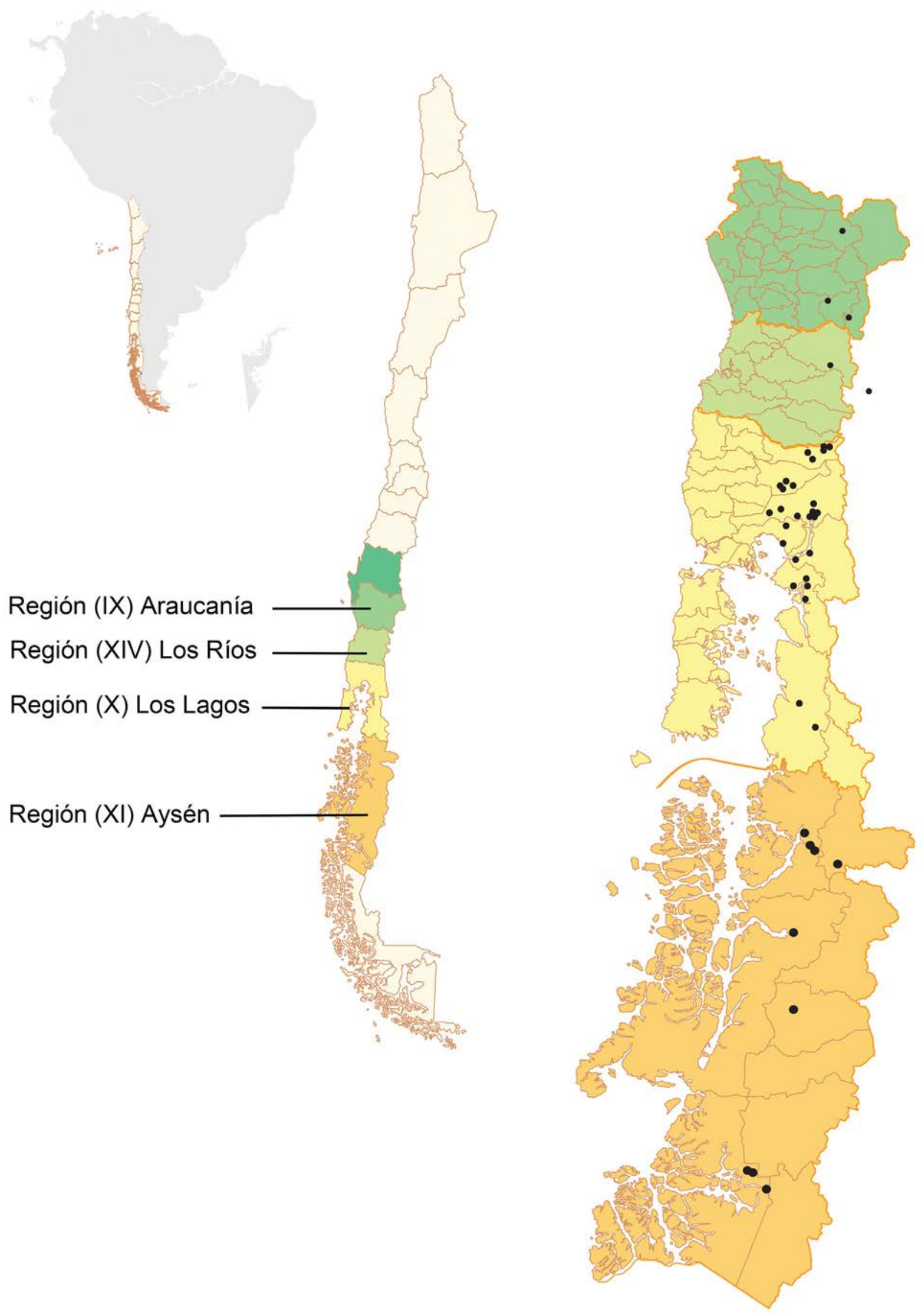

FIGURE 2. Distribution of Araucoderus. 

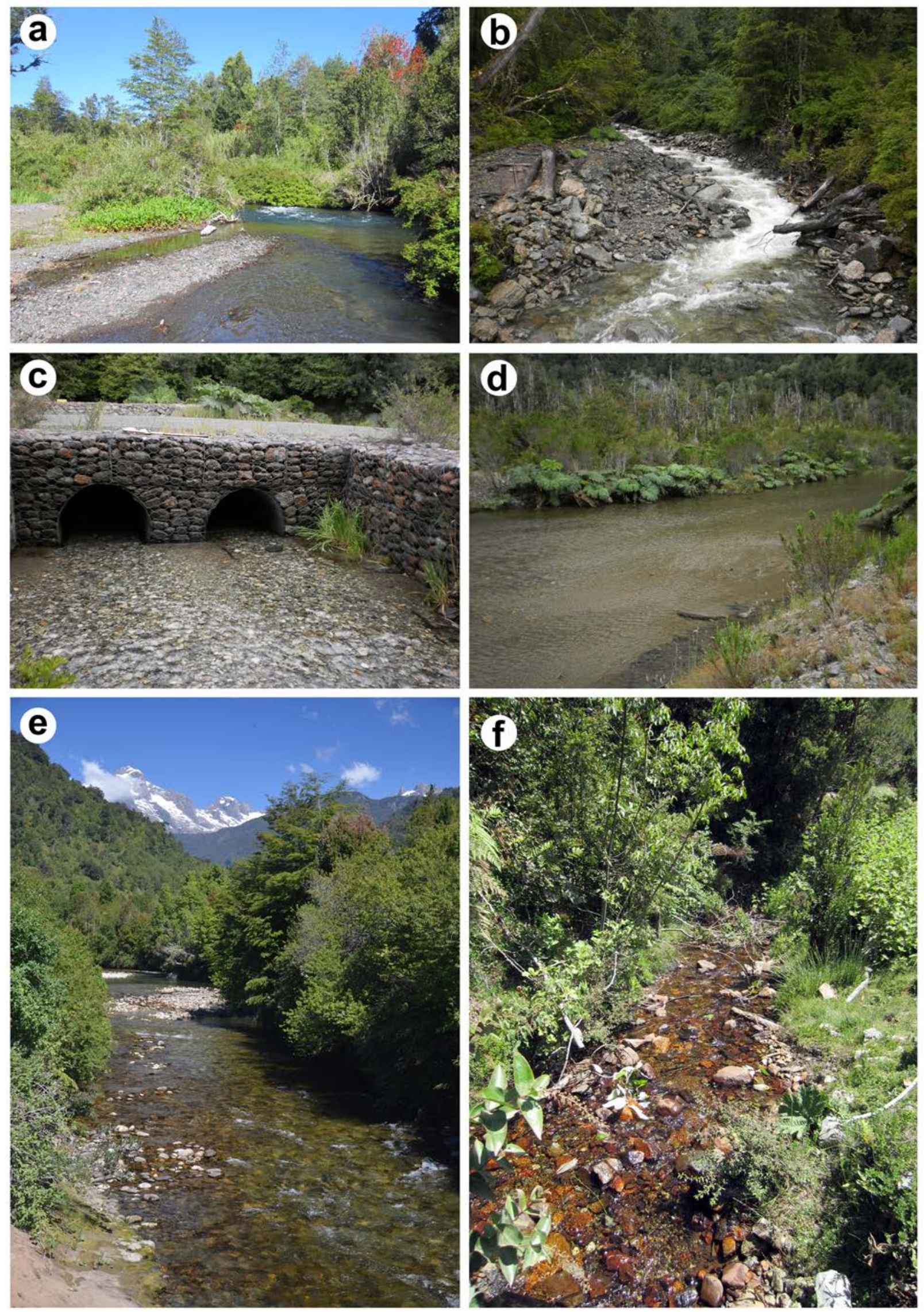

FIGURE 3. Araucoderus habitats in Chile. a. Puyehue N.P., Rio Colorado; b-d. Aysén Province, unnamed creeks; e. Rio Negro near Hornopirén; f. Small creek above Ralún. 
Descriptive format. Diagnoses of the genus and species are provided. Complete descriptions are given for previously unknown life stages and revised descriptions are given for adults. When applicable, sample size is provided before each description with measurements in millimeters presented as a mean followed by a range in parentheses. Measurements: Larval characters refer to instar IV; total length was measured from the anterior-most part of the outer epistomal ridge to the anal aperture. Pupa: total length was from apex of the dorsal apotomal tubercle to the apical-most point of terminal fleshy lobes; respiratory organs were measured from base to apex. Adult: head width was measured at the point of greatest width of the eyes, and head length from the anterior margin of the clypeus to the occiput; leg segments were measured along the dorsal margin; approximate wing length and width were measured at the point of greatest length and width, respectively.

\section{Araucoderus Alexander, 1929}

Araucoderus Alexander, 1929: pp. 228. Type species: Tanyderus gloriosus Alexander, by original designation.

\section{Araucoderus gloriosus (Alexander, 1920)}

Tanyderus gloriosus Alexander, 1920: 13: 5.

Radinoderus gloriosus Alexander, 1927: 189: 9.

Araucoderus gloriosus Alexander, 1929: 1: 228.

Diagnosis. Egg: The only Tanyderidae known in this stage. Larva: cuticle translucent, varying from light grey to tan in color; head orange to brown in color; mandible with three prominent teeth; endocarina (glandular duct) present; postmentum without teeth, flat to convex anteriorly. Pupa: Body elongate, cylindrical; head with tubercles, antennal sheath lying between wing margin and midleg; respiratory organs inconspicuous, undivided and tubular; abdominal segment VIII with digitiform lobes inserted posterolaterally, posterior to spiracle. Male: Cuticle with variegated coloration, light- to dark brown; vestiture light brown; abdominal tergites dark brown, with irregular, pale markings anteriorly on either side of median; wing more than $14 \mathrm{~mm}$ in length, infuscated, with pigment arranged in transverse bands; supernumerary cross-veins absent; male terminalia: with varying degree of rotation, usually 90 degrees, involving segments 7-9; dorsal bridge of paramere with pair of projections anterolaterally; lateromedial element of paramere with small convex anterior lobe bearing 4-6 setiform sensilla, with a dentate mesal lobe set with five dissimilar projections, with a fleshy convex lobe inserted proximally, set with many small trichoid sensilla; dorsomedial element "I-shaped", articulated basally with dorsal bridge, articulated apically with lateral ejaculatory processes of aedeagus; parameral lobe at gonocoxite base reniform in lateral view, with several prominent setiform sensilla inserted subapically; aedeagus trifurcate, with point of trifurcation asymmetrical in dorsal view in some specimens; phallotrema flared slightly. Female: coloration, head, thorax, legs and wings as in male; spermatheca pyriform; ducts uniformly sclerotized, annulated and unpigmented.

Description. See also Alexander (1920). Egg (Figs. 4-6): elongate-ovoid, white, lacking microsculpture; micropile not evident; laid in clusters of 10 or more.

Larva IV instar (Figs. 7-21, 49, 50, 52, 53): Measurements $(\mathrm{n}=3)$, total length $31.34 \mathrm{~mm}(30.43-32.20)$, head width $1.35 \mathrm{~mm}$ (1.33-1.43), head length $1.67 \mathrm{~mm}$ (1.58-1.80). Head capsule ovate, heavily sclerotized anteriorly, dorsoventrally compressed, rounded posteriorly, partially retracted into prothorax; stemmata not apparent; primordial adult eye well-developed; frontoclypeal apotome (frcly ap; Figs. 11, 16) somewhat pentagonal in shape, enclosed by frontal sutures; coronal suture visible medially on posterior $1 / 3$ of the head; mesal endocarina (ec; Fig. $11,16)$ ending medially on anterior clypeal margin; anterior clypeal margin (acm; Fig. 11, 16) composed of two pairs of undifferentiated lobes, medial pair elongate-transverse, lateral pair bulbous; labrum as broad as medial endocarinal lobes combined, broad, fleshy, somewhat dorsoventrally compressed; paraclypeal setae paired (s 3, s 4; Fig. 11); labral sclerite with paired setiform sensilla widely separated (s 2; Fig. 11); palatal brush (pb; Figs. 1214,18) with paired setiform sensilla inserted dorsally (s 1; Fig. 11); antenna (ant; Figs. 10, 12, 18, 20) inserted anterolaterally; base partially shrouded by epicondyle (econ; Figs. 11, 16, 20); two-segmented, basal segment more than twice the length of distal segment; apical segment with at least six inconspicuous, two conspicuous sensilla basiconica inserted apically (aps; Fig. 10); mandibles (md; Figs. 7, 12, 14, 16, 18, 20) well developed, symmetrical, 
pyramidal in shape, scoop-like, with three dentations apically; middle dentation projecting beyond others; oral surface of mandible with well-developed prosthecal brush dorsally, two setiform sensilla ventrally, adoral surface with two sensilla basiconica (s 12, s 13; Fig. 12), anterior condyle well developed; maxillary stipes with one prominent digitiform sensilla anterodorsally (s 21; Fig. 7), with cluster of microtrichia posteroventrally; galea globular (mxg; Figs. 7, 8, 12, 18), partially sclerotized, with three apical setiform sensilla (s 18, s 19, s 20; Figs. 7, 8); lacinia elongate ( $\mathrm{mxl}$; Figs. 7, 8, 12, 18), covered with microtrichia adorally, bearing one seta (s 22; broken in Fig. 9) anterolaterally; maxillary palpus (mxp; Figs. 7-9, 12, 18, 20) unsegmented, cylindrical, slightly longer than wide, set with many sensilla basiconica apically, with apical sensilla globose to digitiform, arranged in whirls; cardo (mxc; Figs. 7, 8, 12, 18) with three setiform sensilla (s 24-s 26; Fig. 8), separated from stipes (mxs; Figs. 8, 12,18 ) by cardostipital ridge; postmentum (Impo; Figs. $8,12,13,18$ ) heavily sclerotized, quadrate basally, with anterior margin straight or slightly emarginated, concave; postgenal bridge (pbr; Fig. 13) complete; prementum (lmpr; Figs. 7, 12-14,18) with saddle-shaped sclerite apically; labial palpus (lmp; Figs. 7, 14) cylindrical, sclerotized, with six digitiform sensilla apically, two almost twice as long as the rest; glossae inconspicuous, (lmg; Fig. 7) conical; hypopharynx (hy; Figs. 7, 13,14) cushion-like, with semicircular sclerite dorsally; pharyngeal filter (ph fi; Fig. 13), housed within pharynx (ph; Fig. 13) well developed, supported by two narrow, sclerotized oral arms originating from hypopharynx. Thoracic segments (pro, mes, met; Fig. 15) equal in length, each bearing ten transverse, equally spaced 1-4 branched setae dorsally, four 2-4 branched setae laterally and six clusters of 1-3 branched setae ventrally; prothorax bearing pair of well-developed spiracles laterally (a spir; Fig. 15), pro- and mesothorax slightly differentiated by tenuous intersegmental constriction; metathorax with circular and pad-like lobes ventrally. Abdominal segments (aI-aVIII \& aA; Fig. 15) with distinct intersegmental constrictions; abdominal segments I-IV with circular lobes ventrally, two simple setae, one bifurcated seta posterolaterally; segment VIII with one pair of posterior spiracles (p spir; Figs. 15, 17, 21), one pair of elongate, annulated filaments extending beyond anal division; filaments with evenly spaced simple setae posteriorly; anal division (aA; Figs. 15, $17,19,21)$ with one pair of elongate, annulated filaments dorsally, one pair of prolegs (pran; Figs. 19, 21) ventrally; prolegs each with elongate, annulated filament inserted dorsoapically, with two rows of retractile hooks apically, basal row with 11-13 hooks, apical row with 12-13 slightly longer hooks; anal papillae (ap; Figs. 15, 17, 19, 21) elongate-digitiform, twice the length of extended prolegs.

TABLE 1. Leg segment measurements for adult males and females of Araucoderus gloriosus.

\begin{tabular}{lllll}
\hline $\begin{array}{l}\text { Adult male } \\
\text { Leg-segment lengths: }\end{array}$ & foreleg & & \\
femur & & midleg & hindleg \\
tibia & & $8.55(5.5-60-9.10)$ & $7.30(6.10-8.50)$ & $7.95(7.00-8.90)$ \\
tibial spur & & $0.31(0.28-0.35)$ & $8.58(7.70-9.85)$ & $10.10(9.10-11.50)$ \\
tarsus & 1 & $8.40(7.80-9.00)$ & $0.32(0.30-0.35)$ & $0.34(0.31-0.39)$ \\
& 2 & $2.58(2.20-2.95)$ & $6.60(5.40-7.30)$ & $6.62(5.50-7.85)$ \\
& 3 & $1.05(1.00-1.10)$ & $2.37(1.80-2.85)$ & $2.36(1.90-2.88)$ \\
& 4 & 0.53 & $1.61(0.97-2.60)$ & $1.27(1.09-1.40)$ \\
& 5 & $0.65(0.58-0.73)$ & $0.68(0.57-0.80)$ & $0.60(0.57-0.66)$ \\
\hline Adult female & & & $0.68(0.58-0.80)$ & $0.58(0.53-0.63)$ \\
femur & & $6.90(6.00-7.80)$ & & \\
tibia & & $9.23(8.20-10.00)$ & $7.73(6.70-9.28)$ & $10.59(9.2-11.52)$ \\
tibial spur & & $0.39(0.33-0.48)$ & $8.92(7.70-9.60)$ & $0.38(0.35-0.42)$ \\
tarsus & 1 & $7.00(6.30-7.40)$ & $0.34(0.28-0.42)$ & $5.73(5.10-6.20)$ \\
& 2 & $2.01(1.00-2.60)$ & $5.60(5.20-5.80)$ & $2.19(2.08-2.30)$ \\
& 3 & $1.26(1.11-1.41)$ & $2.10(1.92-2.25)$ & $1.25(1.13-1.47)$ \\
& 4 & $0.65(0.53-0.72)$ & $1.25(1.16-1.35)$ & $0.68(0.60-0.77)$ \\
& 5 & $0.70(0.61-0.78)$ & $0.65(0.55-0.73)$ & $0.70(0.65-0.81)$
\end{tabular}

Pupa: Male: Measurements $(\mathrm{n}=1)$, total length $18.40 \mathrm{~mm}$, respiratory organ $0.50 \mathrm{~mm}$ (ro; Figs. 23, 25). Body (Figs. 22-25, 51) elongate, cylindrical, with cephalothorax circumference slightly larger than abdomen; mouthparts and thoracic appendages fused to each other and to remainder of cephalothorax. Head with tubercles; dorsal 
apotome (da; Figs. 23, 25) apically with single sensilla (s da; Figs. 22, 24), apotome ventrally abutting anterior margin of face; cephalic sclerite with one seta, sclerite fused anterolaterally to orbital portion of head capsule; antenna (ant; Figs. 22, 24) extending posteriorly to between wing margin and midleg; clypeal apotome with pair of sensilla inserted basally; mouthparts restricted to area anterior of forecoxae; apex of labrum (lbr; Figs. 22, 24) truncate; mandible present, maxilla absent; palpus (plp; Figs. 22, 24) directed obliquely anterolaterally; labella divided medially; sensilla: setae arising from lobes; dorsal apotomals bilobed. Thorax: respiratory organ tubular, with numerous annulations, pores concentrated at apex; legs (pro leg, mes leg, met leg; Figs. 22, 24) directed posteriorly, with tarsi extending posteriorly beyond wing apex, lying parallel to each other; metanotum (met; Fig. 22) undivided medially, haltere (htl; Fig. 22) extended posterolaterally before anterolateral margin of abdominal tergite 2 (aII; Figs. 23, 25). Abdomen: circular in cross-section; tergites I-VIII with setae arranged in transverse rows on sclerotized bands bearing small coniform tubercles; pleurites II-VII with setae arranged in one caudal longitudinal row of three setae and one seta inserted in medial tubercle, adjacent to spiracle; sternites I-VIII with one pair of setae inserted posteriorly; segment VIII with posterolateral fleshy lobes bearing two setae posteriorly to spiracle; Segment IX (aIX; Figs. 23, 25) without setae, a pair of posterior dorsomedial fleshy lobes present; terminal process apically tapered in both sexes. Female: Measurements $(\mathrm{n}=1)$, total length $23.52 \mathrm{~mm}$, respiratory organ $0.57 \mathrm{~mm}$. In other details, same as male.

Male (Figs. 1, 26-29, 32-38, 46): Measurements $(\mathrm{n}=5)$, total length $14.66 \mathrm{~mm}(12.48-17.28)$; head length $1.48 \mathrm{~mm}(1.42-1.56)$; head width $1.22 \mathrm{~mm}$ (1.16-1.30); palpus length $1.54 \mathrm{~mm}$ (segment length average 0.15$0.24-0.57-0.31-0.32)$; clypeus length $0.41 \mathrm{~mm}(0.40-0.44)$; clypeus width $0.37 \mathrm{~mm}(0.35-0.39)$; wing length $15.82 \mathrm{~mm}$ (14.14-18.08); wing width $3.75 \mathrm{~mm}$ (3.35-4.05). Head with mandible and maxilla poorly developed (md, mx; Fig. 27); antenna with scape and pedicel glabrous, each with several setiform sensilla inserted distally; scape cylindrical (scp; Figs. 27, 28), slightly larger than pedicel; pedicel spherical (ped; Figs. 27, 28); flagellomeres numbering 15 , all elongate, more than 3 times longer than wide, cylindrical; f 1 slightly longer than $\mathrm{f} 2$; f $3-14$ each with 4-5 trichoid sensilla inserted near base of flagellomere, arranged concentrically, as long or longer than flagellomere; f 1-15 each with several basiconic sensilla throughout; f 15 with 3 trichoid sensilla inserted apically. Cervical sclerites more than two times longer than wide. Wing (Figs. 30, 31) held away from body at angle in live resting individual; infuscate, with three transverse bands of pigment, basal-most band extending from $\mathrm{h}$ vein to posterior wing margin and slightly beyond proximal area of anal loop, medial band extending from midlength of Sc vein to posterior edge of $\mathrm{C}$ vein at distal end of anal lobe, passing through $\mathrm{r}-\mathrm{m}$ and $\mathrm{m}-\mathrm{cu}$; apical-most band touching $C$ vein on both sides of wing, appearing as an " $X$ " from anterior margin of wing to apices of $R_{4}, M_{2}$; costal cell yellow in color; cell $r_{1}$ with two isolated, irregular, infuscated spots, one midlength of vein Rs and a second spot at cell apice; other spots, when present, variable among individuals. Halteres pale yellow at base, dark at apex. Legs light to dark brown, darker at both ends: Tibial spurs 0-2-2, symmetrical. Abdomen: segment I less than half as long as next segment, causing abdomen to droop ventrally; tergites dark brown with large irregular pale yellow markings basally on either side of medial line, each with dark yellow ovoid pattern centrally, basally. Terminalia (Figs. 32-38) with torsion of segments 7-9 variable from 0 to 180 degrees; gonocoxites (goncx; Figs. 32, 33, 3638) narrowly contiguous at base (Fig. 33), divergent from each other at origin, each nearly cylindrical, tapering slightly toward apex; setiform sensilla or setae alveoli distributed over dorsal, ventral and lateral surface, absent mesally; gonostylus (gonst; Figs. 32, 33, 36) cylindrical, about 2/3 length of gonocoxite, slightly tapering at apex, curved medially, with prominent setae alveoli evenly distributed over surface, hirsute apically; hypandrium not evident; epandrium (epand; Figs. 32, 36-38) slightly wider than long, with setae alveoli distributed over entire surface; proctiger recessed anteriorly, nested within epandrium; cercus (cerc; Figs. 37, 38) inconspicuous, unmodified, setose; paramere subdivided into dorsal bridge ( $\mathrm{pm} \mathrm{db}$; Fig. 37), lateromedial element (pm lme; Figs. 36-38), dorsomedial element (pm dme; Figs. 37, 38) and parameral lobe at gonocoxite base (pm gbl; Figs. 32, 33, 37,38 ); dorsal bridge arch-shaped, with a pair of small projections anterolaterally; lateromedial element broadly interconnected with gonocoxite anteromesally, divided into three prominent lobes, anterior lobe small, convex, with 4-6 setiform sensilla, mesal lobe dentate, with five dissimilar projections, with fleshy convex lobe proximally bearing many small trichoid sensilla; dorsomedial element "I-shaped", articulated basally with dorsal bridge, articulated apically with lateral ejaculatory processes of aedeagus; parameral lobe at gonocoxite base reniform in lateral view, with several prominent setiform sensilla inserted subapically, many smaller trichoid sensilla inserted on entire surface; ejaculatory apodeme (ej apod; Figs. 32-35, 38) extending anteriorly to abdominal segment 7 terminus, laterally compressed, clavate at base, with surface corrugated; sperm sac (spm sac; Figs. 34, 35) balloon- 
like, surrounded by aedeagus posteriorly, attached to ejaculatory apodeme anteriorly; aedeagus (aed; Figs. 32-38) narrowed abruptly at base, trifurcated at midlength; point of trifurcation asymmetrical in dorsoventral view; phallotrema flared slightly, placed between cerci when at rest; testes (tes; Figs. 32-35, 38) pyriform, rounded anteriorly, differentiated from vasa deferentia only by width, shape; vasa deferentia (v d; Figs. 34, 35), testes rugose in appearance; ejaculatory duct (ej dt; Figs. 34, 35) lightly sclerotized, entering sperm sac through ejaculatory apodeme foramen.
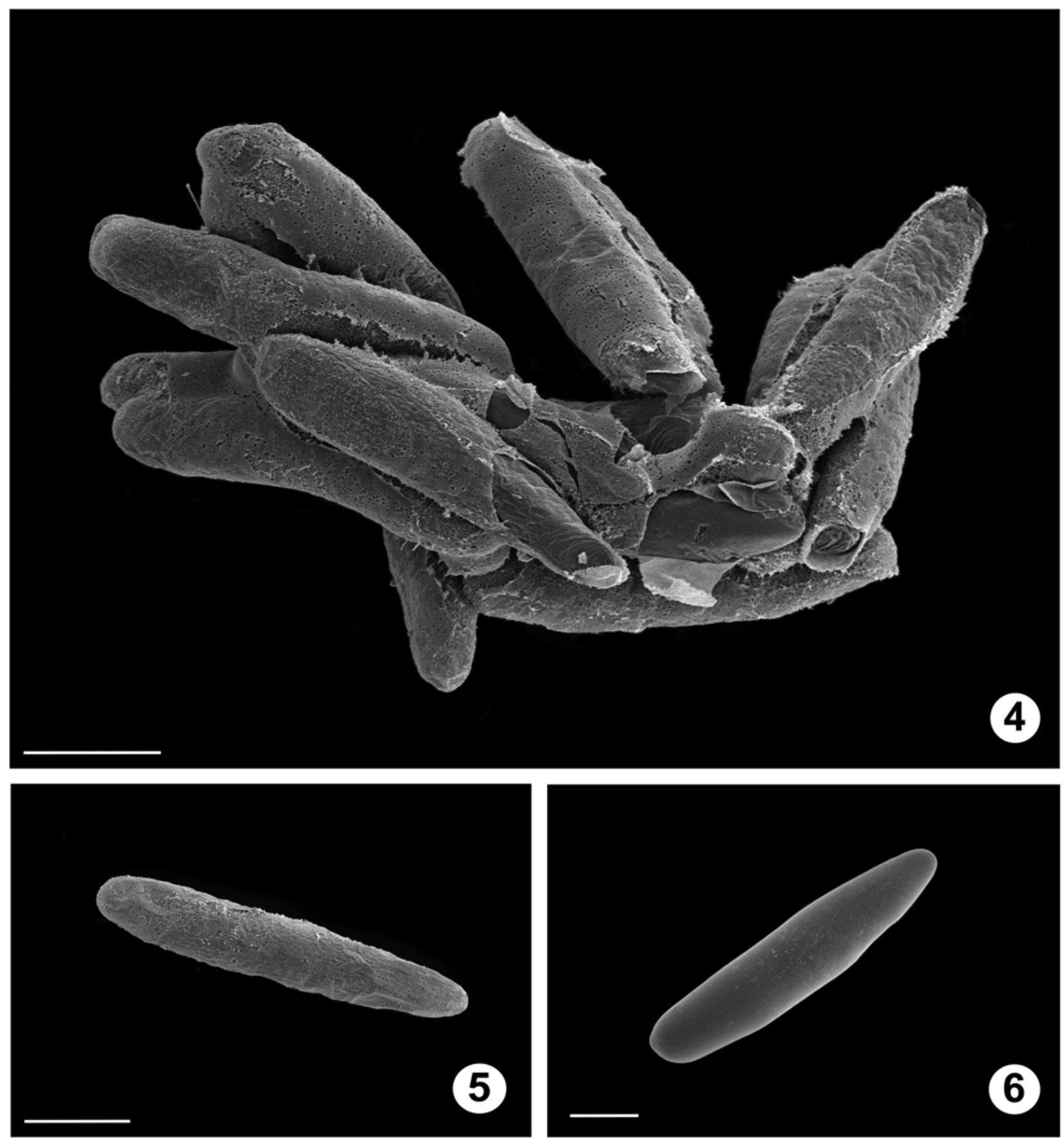

FIGURES 4-6. Araucoderus gloriosus eggs. 4. Egg cluster. 5. Single egg with chorion. 6. Single egg without chorion. Scale bars $=0.20 \mathrm{~mm}$.

Female (Figs. 39-45): Measurements $(\mathrm{n}=3)$, total length $21.02 \mathrm{~mm}(19.20-24.48)$; head length $1.51 \mathrm{~mm}$ (1.25-1.72); head width $1.23 \mathrm{~mm}$ (1.14-1.38); clypeus length 0.47 (0.37-0.55); clypeus width 0.40 (0.35-0.47); palpus length $1.80 \mathrm{~mm}$ (palpomere length average $0.11-0.34-0.66-0.32-0.36)$; wing length $18.56 \mathrm{~mm}(16.15-$ 22.08); wing width $4.92 \mathrm{~mm}$ (4.00-5.96). Head with eyes as in male; mandible and maxilla twice as long as wide; 
antenna as in male. Wing as in male. Terminalia with posterior margin of sternite VIII broadly bilobate, strongly emarginate medially, emargination with "square U" shape; genital fork (gen fk; Figs. 40, 42, 43, 44) transversely elongated posteriorly, more than eight times longer than wide, "Y"-shaped medially, acuminate anteriorly; spermatheca numbering three; corpora ovoid, with visible necks; spermathecal ducts annulated and uniformly sclerotized, more than twice as long as corpora, unpigmented.

Distribution. Araucoderus is restricted to Patagonia. All confirmed records lie between latitudes $38^{\circ} 31^{\prime} \mathrm{S}$ and $48^{\circ} 02^{\prime} \mathrm{S}$, ranging in altitude from 2 to 1700 masl. A survey of the central regions of Chile yielded no specimens. There is one published Araucoderus record from Argentina (Alexander, 1959).

Type material. Chile. Región XI (Aysén): Holotype ${ }^{\top}$ : Chile Rio Aysén, approximately, coll. P. Dusen, no date given; the specimen is deposited in two museums. Naturhistoriska Riksmuseet Stockholm, Sweden (NHRM) and the USNM. The latter possesses only the wing, antenna and middle leg. Only the USNM material was examined during the current study.

Material examined. See Figure 2. Chile. Región IX (Araucanía): Estero el Rosario @ confluence with Estero la Cascada 39²3'12.93”S 7141'46.27’W, 710masl, 3.x.2007, coll. G.W. Courtney, [2L]; Villarica N.R. Río

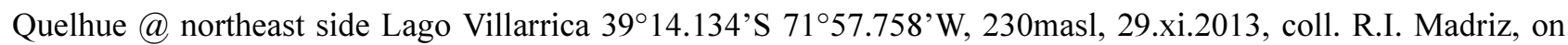

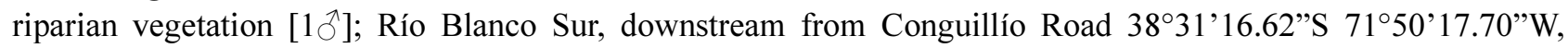
601masl, 19.xii.2013, coll. G.R. Curler, riparian vegetation [1]․ Región XIV (Los Ríos): Estero Truful @ Hwy 203 crossing 3949’29.97”S 7159’53.63”W, 230masl, 4.xii.2013, coll. R.I. Madriz [3 $\left.{ }^{\Uparrow}\right]$ ]. Región X (Los Lagos): Casa Pangue Llanquihue, 4.xii.1926, coll. E.S. Shannon [1 ${ }^{\lambda}$; Ensenada, Llanquihue, 2.i.1937, coll. E.C. Reed [1§]; Llanquihue Hornohuinco, xii.1968, coll. L.E. Pena [1 $\left.{ }^{\top}\right]$; Lago Puyehue southeast shore, 10.ii.1978, coll. W.N. Mathis [1 ${ }^{\lambda}$ ]; Osorno Prov. Laguna el Espejo, 7.ii.1978, col. W.N. Mathis [1 $\left.{ }^{\Uparrow}\right]$; Palena Río Amarillo ca. $28 \mathrm{~km}$ southeast Chaitén, 23.i.1987, coll. C.M. \& O.S. Flint, Jr., [1 $\left.{ }^{\Im}\right]$; Llanquihue Prov. Río del Este @ Río

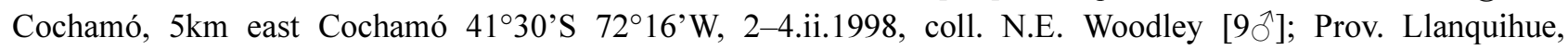
\{Cayutue\} near Ralún $41^{\circ} 14^{\prime} \mathrm{S} 72^{\circ} 16^{\prime} \mathrm{W}, 12.1 i .1998$, coll. N.E. Woodley [2`]; Puyehue N.P., Río Anticura above Salto Río Anticura, 4040’16.14”S 72॰10’12.88”W, 395masl, 4.xii.2008 coll. G.W. Courtney [1 $\left.{ }^{`}\right]$; Puyehue N.P., Río Anticura above Salto Río Anticura, 4040’16.14”S 72¹0’12.88”W, 395masl, 6.xii.2013, coll. R.I.

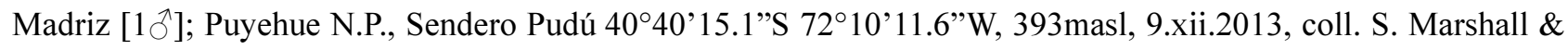
G.W. Courtney [1 $\left.{ }^{\Uparrow}\right]$; Río Blanco @ Puente Río Blanco Arenales 4103’04.6”S 72³9’46.8”W, 69masl, 9\&13.xii.2013, coll. R.I. Madriz \& G.W. Courtney [2L, 1P, 2ð̂, 1 + ]; Puyehue N.P., Río Colorado @ Puente Arauco Sendero de Chile 40 40'15.5”S 72॰08'43.9”W, 467masl, 10.xii.2013, coll. R.I. Madriz, G.W. Courtney \& G.R. Curler [10L, 1P, 13ð,1]; Small creek $\approx 7 \mathrm{~km}$ above Ralún on road to Cayetué $41^{\circ} 19^{\prime} 50.7^{\prime}$ 'S $72^{\circ} 16^{\prime} 30.1^{\prime}$ W, 240masl, 12.xii.2013, coll. R.I. Madriz \& G.W. Courtney [6L, 5 đ]; Cayetué Road, small creek $\approx 5.5 \mathrm{k}$ above Ralún 41 ${ }^{\circ} 20^{\prime} 20.89 " \mathrm{~S} 72^{\circ} 16^{\prime} 23.40^{\prime \prime} \mathrm{W}, 150 \mathrm{masl}$, 13.xii.2013, coll. G.W. Courtney, riffle kick sample [5L]; Río Pedregoso

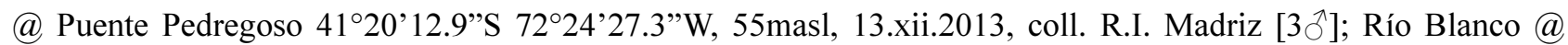
Puente Río Blanco, 4058’36.5”S 72³5’01.9”W, 242masl, 14.xii.2013, coll. R.I. Madriz [2〕]; Río Blanco @ Puente Río Blanco 4100’30.2”S 72³4’13.9”W, 330masl, 14.xii.2013, coll. R.I. Madriz [2`]; Río Llaquepe @ Puente Llaquepe 41 43'43.9'S 72²8'51.4”W, 33masl, 15.xii.2013, coll. R.I. Madriz [1 + ]; Río Negro Chaqueihua $0.5 \mathrm{~km}$ N of intersection to Hornopirén N.P. $41^{\circ} 55^{\prime} 38.2^{\prime \prime S} 72^{\circ} 25^{\prime} 19.6^{\prime \prime} \mathrm{W}, 39$ masl, 15.xii.2013, coll. R.I. Madriz \&

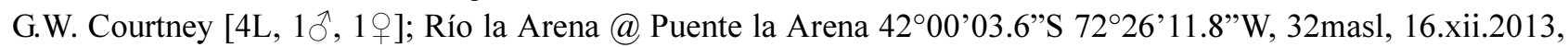
coll. R.I. Madriz [1P, 1 ${ }^{\Uparrow}$ ]; Río Santa Juanita @ Puente Santa Juanita 4207’38.1”S 72²7’54.8”W, 32masl,

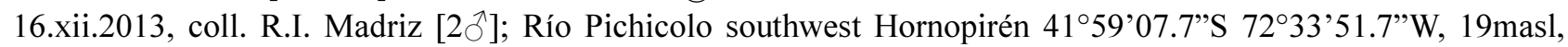
17.xii.2013, coll. G.W. Courtney [3L]; Región XI (Aysén): Creek crossing @ highway 7 46ำ14'46.0”'S $72^{\circ} 47^{\prime} 31.9^{\prime} \mathrm{W}, 273 \mathrm{masl}, 27 . x i i .2013$, coll. R.I. Madriz [1 ${ }^{`}$ ]; West of intersection to Caleta Tortel $47^{\circ} 46^{\prime} 48.1^{\prime \prime} \mathrm{S}$ 73¹9’00.2”W, 51masl, 26.xii.2013, coll. R.I. Madriz [11 ${ }^{\top}, 3$, $]$; Creek @ road to Ventisquero Mont 6km south of intersection to Villa O'Higgins $48^{\circ} 02^{\prime} 58.7^{\prime}$ 'S 7307'52.7”'W, 74masl, 25.xii.2013, coll. R.I. Madriz [1 $\left.{ }^{\jmath}\right]$.

Natural history. Habitat. All larvae were collected in water temperatures from 4 to $11^{\circ} \mathrm{C}$, with most sites closer to $4^{\circ} \mathrm{C}$. The riparian zone of inhabited streams varied from dense forest to sites with little to no riparian cover (Fig. 3). Collection sites typically had coarse gravel to rocky substrata, with submerged wood either sparse or abundant. Some sites had diagonal and mid-channel gravel bars. Substrata often contained abundant detritus. Stomach contents of captured specimens suggest Araucoderus larvae feed on fine particulate organic matter. 

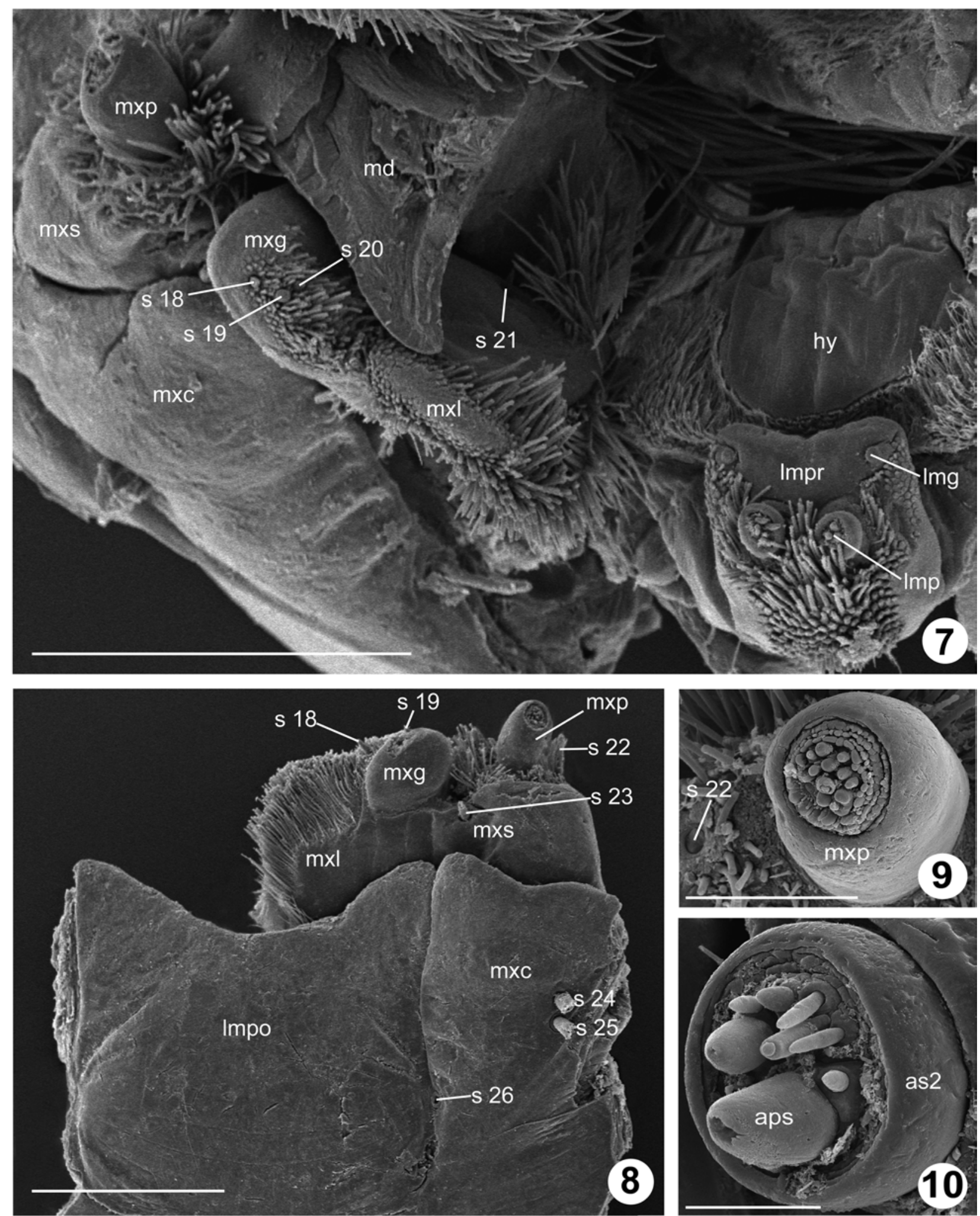

FIGURES 7-10. Head capsule parts of Araucoderus sp. 7. Primary right mouth parts frontal view with apical part of the mandible removed. 8. Left mouth parts ventral view (some labeled setae broken or missing). 9. Right maxillary palpus with seta broken off. 10. Left antenna posterodorsal oblique view. Abbreviations: aps = apical sensillum; as2 = antennal segment \#2; hy = hypopharynx; lmg = glossae; $1 \mathrm{mp}=$ labial palpus; $1 \mathrm{mpo}=$ postmentum; $1 \mathrm{mpr}=$ prementum; $\mathrm{md}=$ mandible; $\mathrm{mxc}=$ cardo; $\mathrm{mxg}=$ galea; $\mathrm{mxl}=$ lacinia; $\mathrm{mxp}=$ maxillary palpus; $\mathrm{mxs}=$ stipes; $\mathrm{s} 18-26=$ seta, \#18-\#26. Scale bar $7=0.20 \mathrm{~mm}(7 \& 8), 0.05$ $\mathrm{mm}(9), 0.20 \mathrm{~mm}(10)$ 

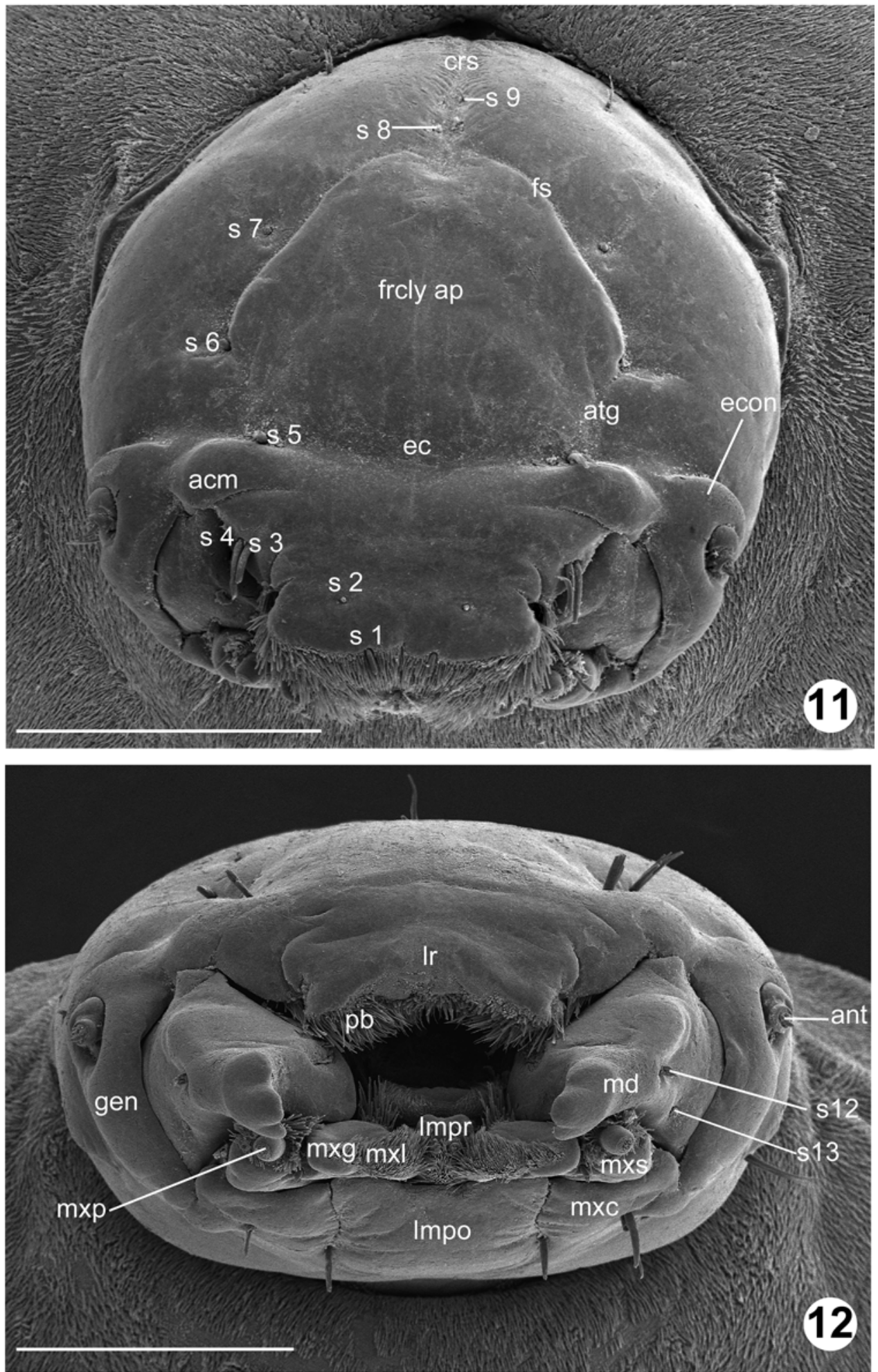

FIGURES 11-12. Head capsule of Araucoderus sp. 11. Anterodorsal view. 12. Frontal view. Abbreviations: acm = anterior clypeal margin; ant = antenna; atg = anterior tentorial groove; $\mathrm{crs}=$ coronal sulcus; ec = endocarina; econ = epicondyle; frcly ap = frontoclypeal apotome; $\mathrm{fs}=$ frontal suture; gen = gena; $1 \mathrm{mpo}=$ postmentum; $\operatorname{lmpr}=$ prementum; $\operatorname{lr}=$ labrum; $\mathrm{md}=$ mandible; $\mathrm{mxc}=$ cardo; $\mathrm{mxg}=$ galea; $\mathrm{mxl}=$ lacinia; $\mathrm{mxp}$; maxillary palpus; $\mathrm{mxs}=$ stipes; $\mathrm{pb}=$ palatal brush; $\mathrm{s} 12-13=$ seta, \#12-\#13. Scale bars $=0.50 \mathrm{~mm}$ 

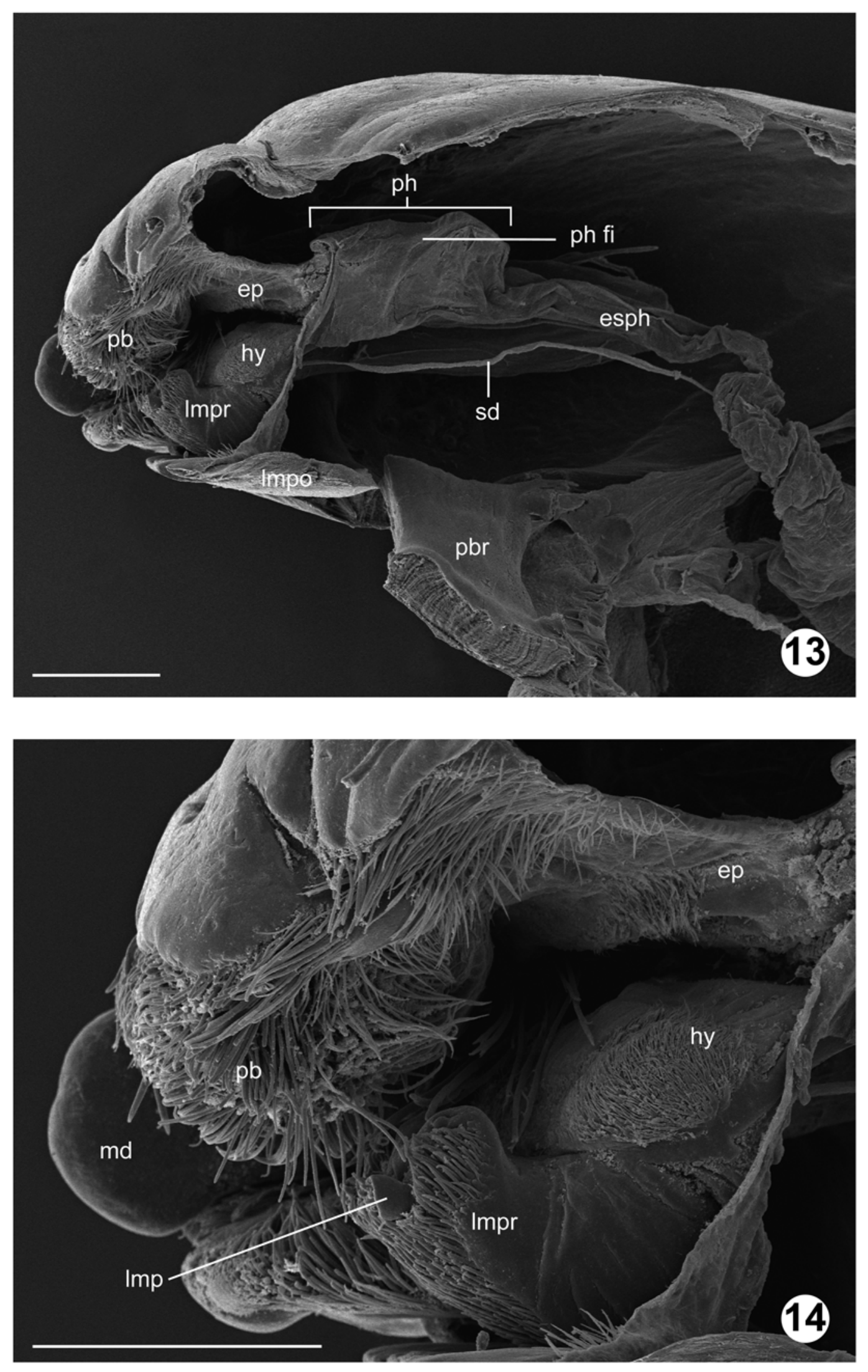

FIGURES 13-14. Araucoderus sp. 4th instar larva saggital cross section of head capsule. 13. Complete head capsule. 14. Mouth parts close up. Abbreviations: ep = epipharynx; esph = esophagus; hy = hypopharynx; $\operatorname{lmp}=$ labial palpus; lmpo = postmentum; lmpr = prementum; $\mathrm{md}=$ mandible; $\mathrm{pb}=$ palatal brush; $\mathrm{pbr}=$ postgenal bridge; $\mathrm{ph}=$ pharynx; $\mathrm{ph} \mathrm{fi}=$ pharyngeal filter; sd = salivary duct. Scale bars $=0.20 \mathrm{~mm}$ 

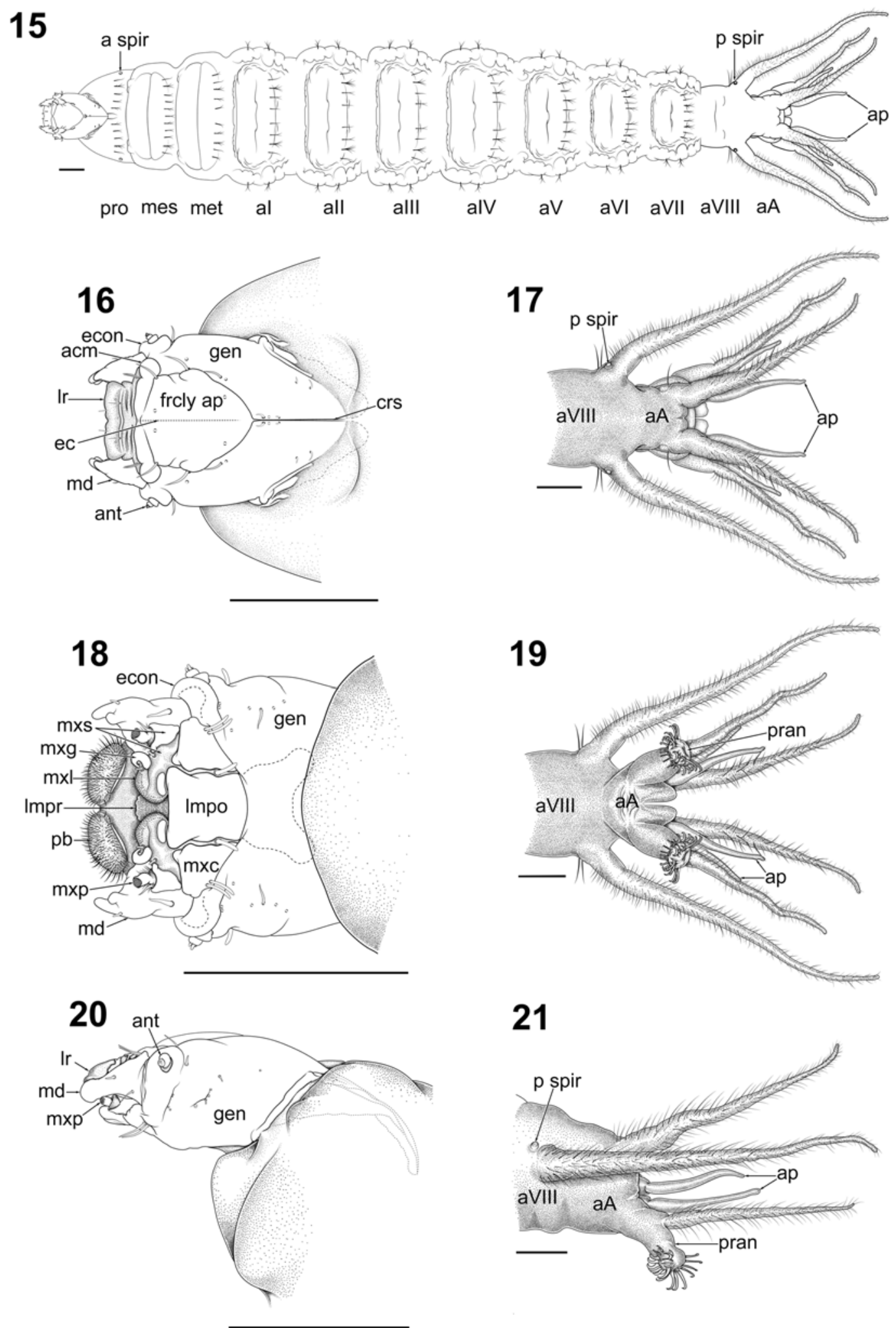

FIGURES 15-21. Araucoderus $s p$. 4th instar larva. 15. Habitus dorsal view. 16. Head capsule dorsal view. 17. Anal division dorsal view. 18. Head capsule ventral view. 19. Anal division ventral view. 20. Head capsule lateral view. 21. Anal division lateral view. Abbreviations: a spir = anterior spiracle; $\mathrm{acm}=$ anterior clypeal margin; ant = antenna; $\mathrm{aA}=$ anal division; ap = anal papillae; $\mathrm{crs}=$ coronal sulcus; ec = endocarina; econ = epicondyle; frcly ap = frontoclypeal apotome; gen = gena; $1 \mathrm{mpr}=$ prementum; lmpo = postmentum; $1 \mathrm{r}=$ labrum; $\mathrm{md}=$ mandible; mes = mesothorax; met = metathorax; $\mathrm{mxc}=\mathrm{cardo} ; \mathrm{mxg}=$ galea; $\mathrm{mxl}=$ lacinia; $\mathrm{mxp}$; maxillary palpus; $\mathrm{mxs}=$ stipes; $\mathrm{p}$ spir = posterior spiracle; $\mathrm{pb}=$ palatal brush; pran = anal proleg; pro $=$ prothorax; $\mathrm{aI}-\mathrm{aVIII}=$ abdominal segments I-VIII. Scale bars $=1.00 \mathrm{~mm}$. 


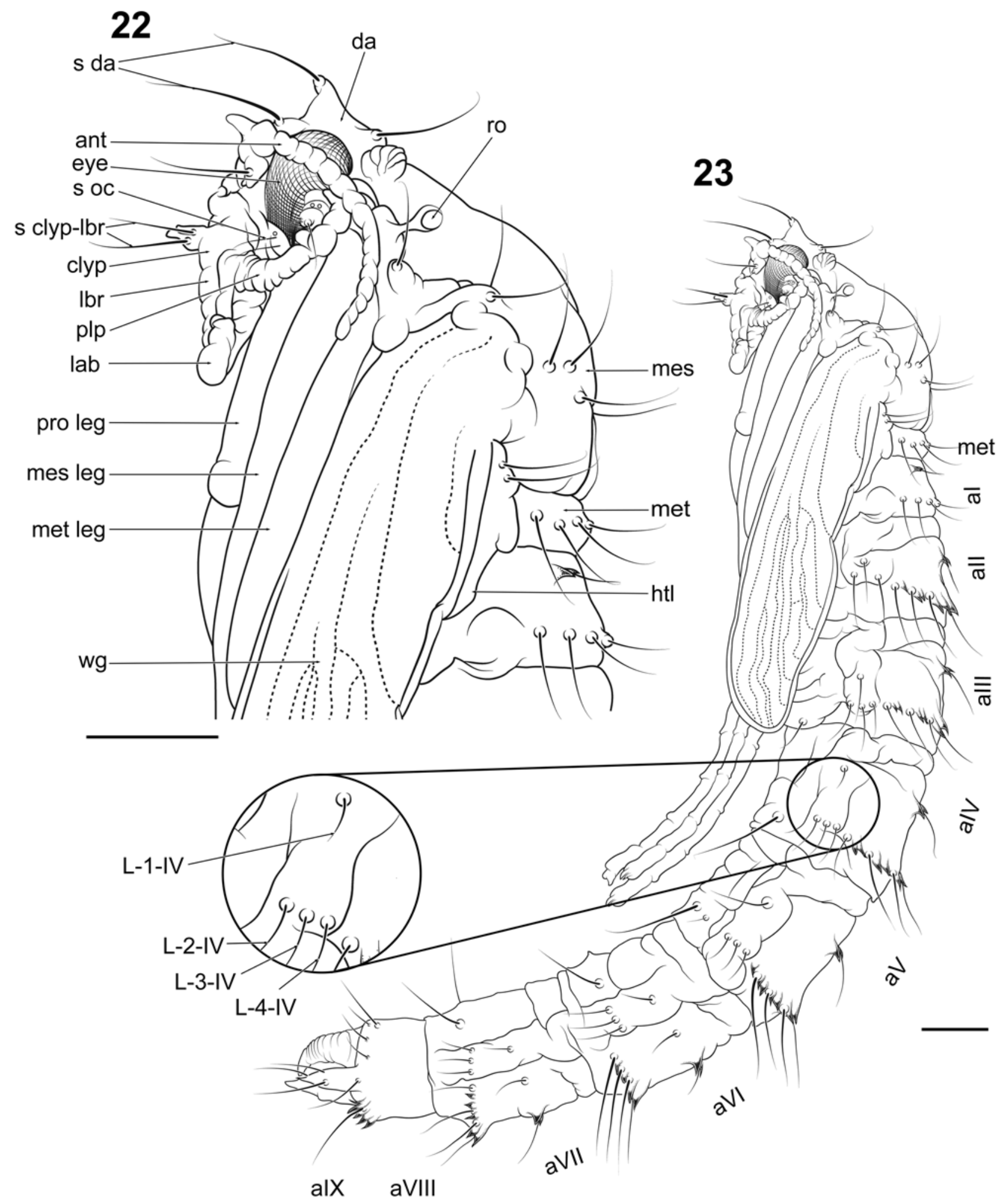

FIGURES 22-23. Araucoderus gloriosus (Alexander) pupa. 22. Habitus lateral view. 23. Head \& thorax lateral view. Abbreviations: aI-aIX = abdominal segments; ant = antenna; clyp = clypeus; da = dorsal apotome; eye = eye; htl = haltere; met leg = hind leg; lab = labium; lbr = labrum; mes = mesonotum; mes leg = mid leg; met = metanotum; plp = palpus ; pro leg = foreleg; ro = respiratory organ; s clyp-lbr = clypeal-labral setae; $\mathrm{s} \mathrm{da}=$ dorsal apotomal setae; $\mathrm{s} \mathrm{oc}=$ ocular setae; $\mathrm{wg}=$ wing; 1 $1-4-\mathrm{IV}=$ segment 4 sensilla $\# 1-\# 4$. Scale bars $=0.01 \mathrm{~mm}$. 


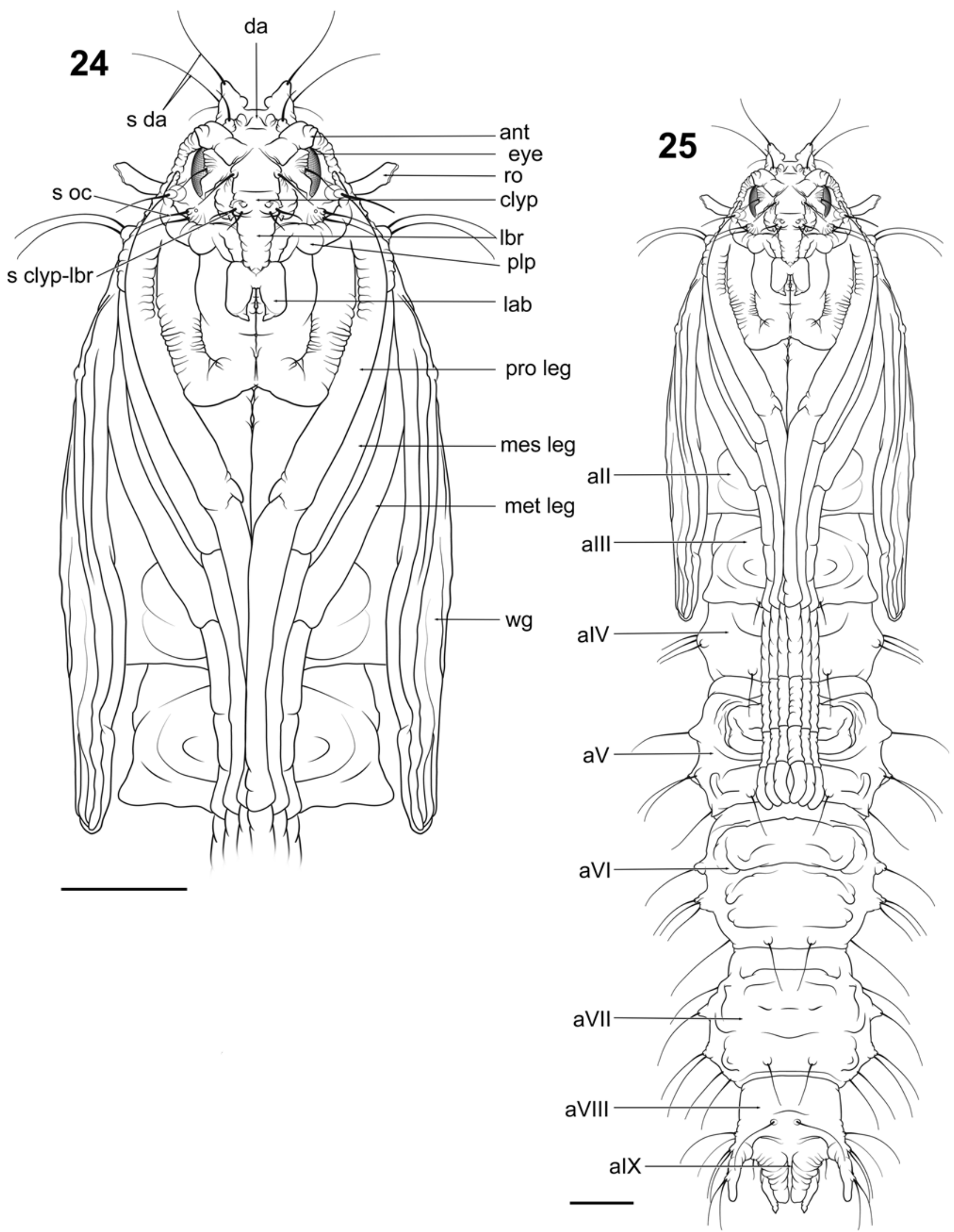

FIGURES 24-25. Araucoderus gloriosus (Alexander) pupa. 24. Habitus ventral view. 25. Head \& thorax ventral view. Abbreviations: ant $=$ antenna; clyp = clypeus; $\mathrm{da}=$ dorsal apotome; eye = eye; lab = labium; lbr = labrum; mes leg = mid leg; met leg = hind leg; plp = palpus ; pro leg = foreleg; ro = respiratory organ; s clyp-lbr = clypeal-labral setae; $\mathrm{s}$ da $=\mathrm{dprsa}$; apotomal setae; $\mathrm{s} \mathrm{oc}=$ ocular setae; wg = wing; aII-aIX = abdominal segments. Scale bars $=0.01 \mathrm{~mm}$ 

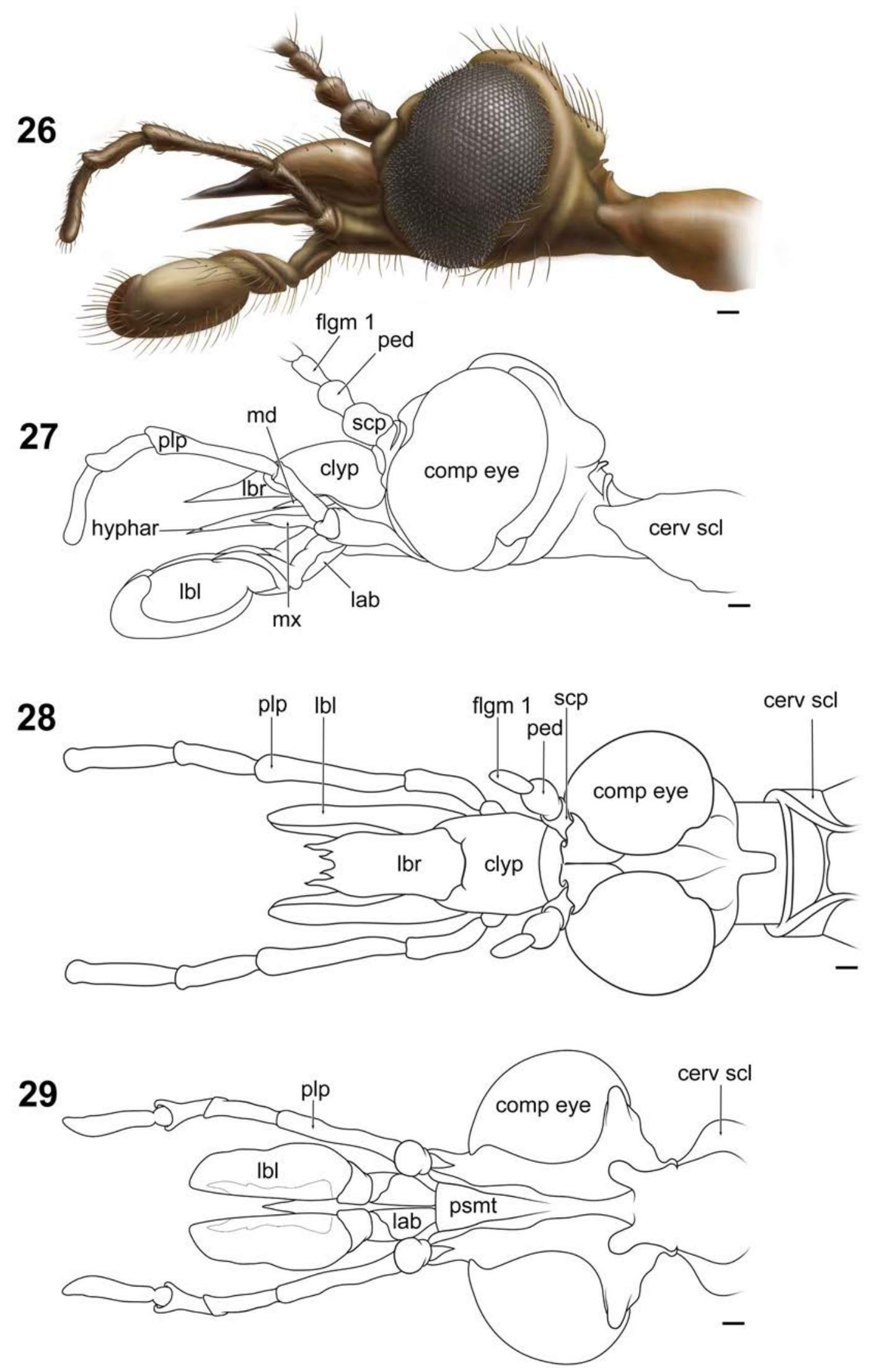

FIGURES 26-29. Araucoderus gloriosus (Alexander) adult male head capsule with flagellomeres 2-17 removed. 26. Lateral view. 27. Lateral view. 28. Dorsal view. 29. Ventral view. Abbreviations: cerv scl = cervical sclerite; clyp = clypeus; comp eye = compound eye; flgm 1 = flagellomere \#1; hyphar = hypopharynx; lab = labium; lbl = labellum; lbr = labrum; $\mathrm{md}=\mathrm{mandible}$; $\mathrm{mx}=$ maxilla; ped $=$ pedicel; plp $=$ palpus; $p s t m=$ postmentum; $\mathrm{scp}=$ scape. Scale bars $=0.10 \mathrm{~mm}$ 

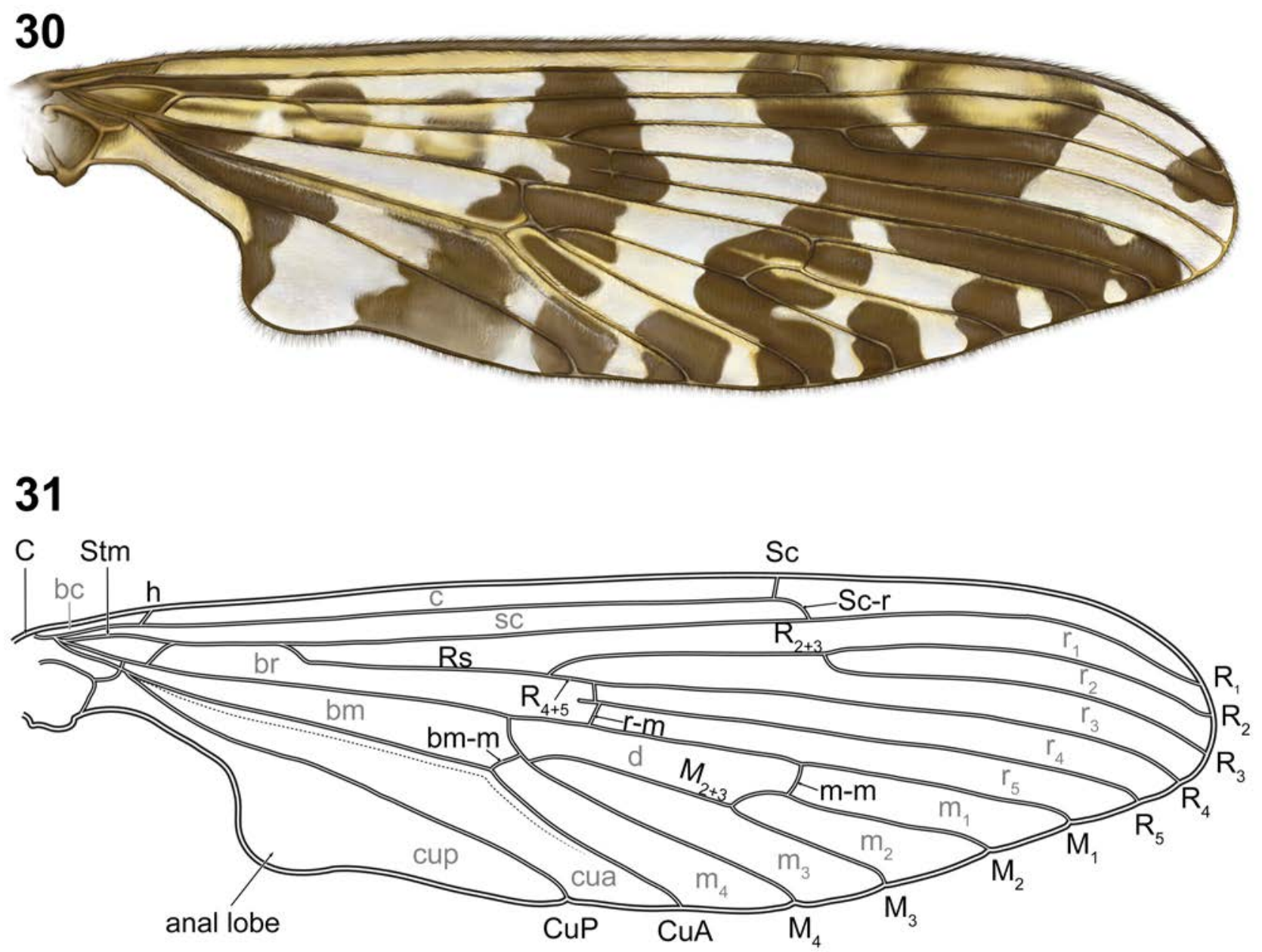

FIGURES 30-31. Araucoderus gloriosus (Alexander) wing. 30. With pigmentation. 31. Without pigmentation, veins and cells indicated. Abbreviations: anal lobe $=$ anal lobe; $b c=$ basal costal cell; $b m=$ basal medial cell; $b m-m=$ basal medial-medial crossvein; $\mathrm{br}=$ basal radial cell; $\mathrm{c}=$ costal cell; $\mathrm{C}=$ costal vein; cua $=$ anterior cubital cell; $\mathrm{CuA}=$ anterior branch of cubitus vein; cup = posterior cubital cell; $\mathrm{CuP}=$ posterior branch of cubitus vein; $\mathrm{d}=$ discal cell; $\mathrm{h}=$ humeral crossvein; $\mathrm{m}-\mathrm{m}=\mathrm{medial}$ crossvein; $\mathrm{m}_{1}, \mathrm{~m}_{2}, \mathrm{~m}_{3}, \mathrm{~m}_{4}=$ medial cells; $\mathrm{M}_{1}, \mathrm{M}_{2}, \mathrm{M}_{3}, \mathrm{M}_{4}=$ distal branches of medial vein; $\mathrm{MA}=$ anterior branch of medial vein; $\mathrm{r}-\mathrm{m}=$ radial-medial crossvein; $\mathrm{r}_{1}, \mathrm{r}_{2}, \mathrm{r}_{3}, \mathrm{r}_{4}, \mathrm{r}_{5}=$ radial cells; $\mathrm{R}_{1}, \mathrm{R}_{2}, \mathrm{R}_{3}, \mathrm{R}_{4}, \mathrm{R}_{5}=$ distal branches of radius; $\mathrm{Rs}=$ radial sector vein; $\mathrm{sc}-\mathrm{r}=$ subcostal-radial crossvein; $\mathrm{sc}=$ subcostal cell; $\mathrm{Sc}=$ subcosta vein; $\mathrm{Stm}=$ stem vein.

Captive reared larvae became increasingly active a few days prior to pupation, possibly in search of a suitable pupation site. During morning field collections, larvae were sometimes found crawling on the surface of the marginal substrate. Captive individuals became increasingly active during the morning from 0900-1100hrs. In the field, mature larvae were often found beside a rock that was much larger than the larva, sometimes partially buried in the contiguous sandy substrata (Fig. 52).

Resting behavior of adults. In both the field and laboratory, we observed no A. gloriosus adults resting on horizontal surfaces. The few specimens found on sloped surfaces used their tibial spurs as the main point of support. In the field, most adults were found on the underside of broad leaves in the riparian zone or among exposed roots of undercut river banks in the same general location as larvae (Figs. 46-53), sometimes more than 10 $\mathrm{m}$ away from the stream. Among the most common resting sites were the undersides of leaves of Gunnera sp., Chusquea sp., or Fuschia sp. A few individuals of both sexes were found under bridges, between the gabions armoring bridge abutments or on vertical surfaces of rocks and logs. A morphological study of the tarsal segments in A. gloriosus revealed similarities to those of Mischoderus Handlirsch, 1909 (Friedemann et al. 2014) and suggests that specific tarsal attachment devices are not required for the observed resting behavior in Araucoderus and Mischoderus. 
32
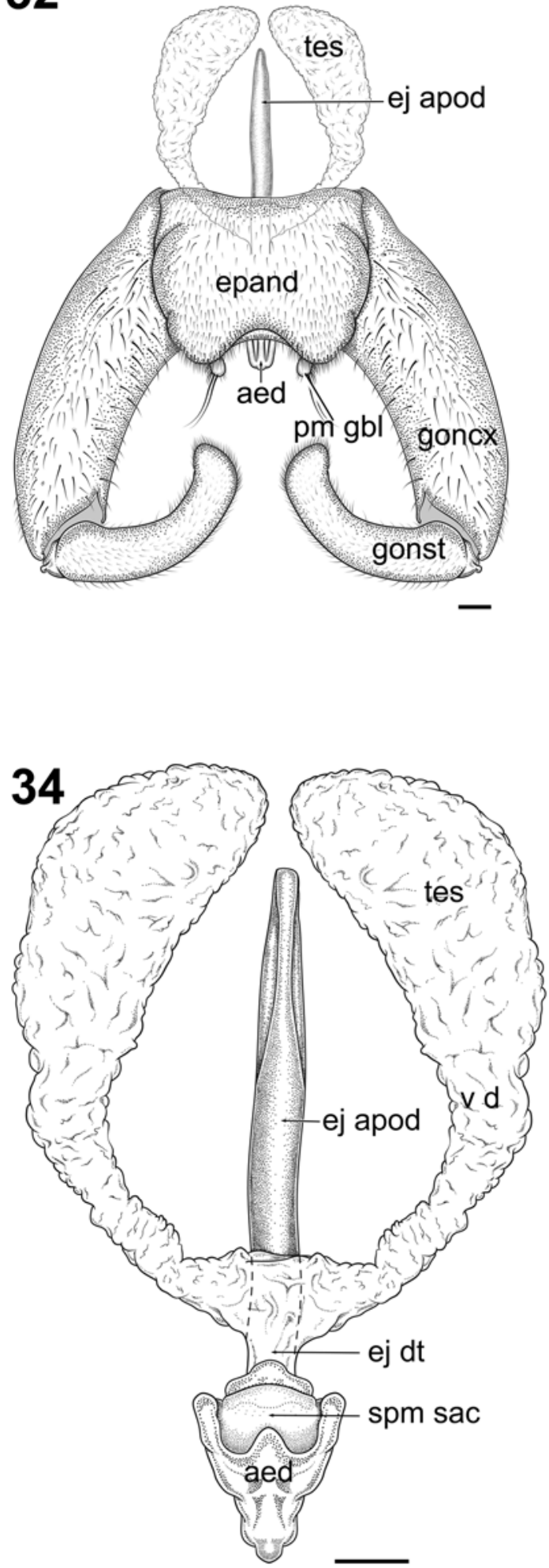

33

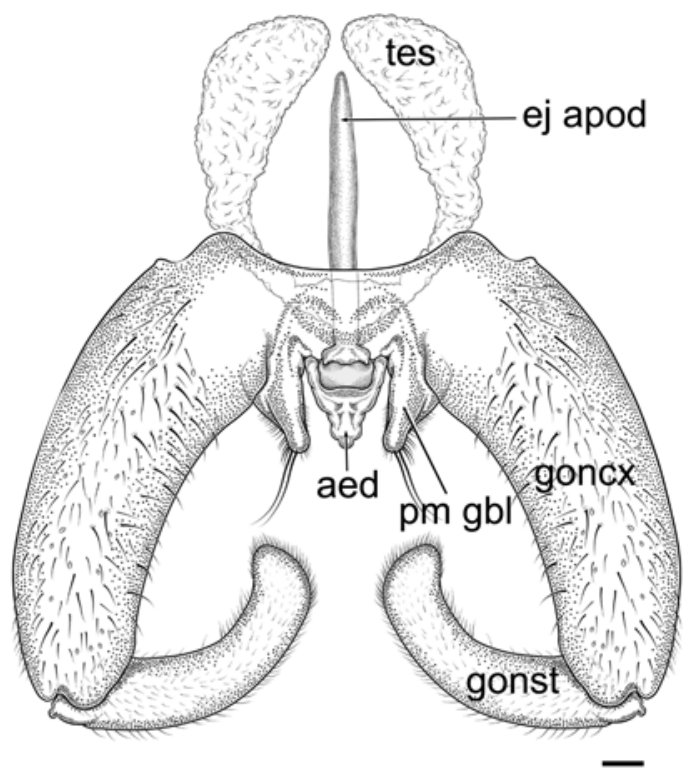

\section{5}

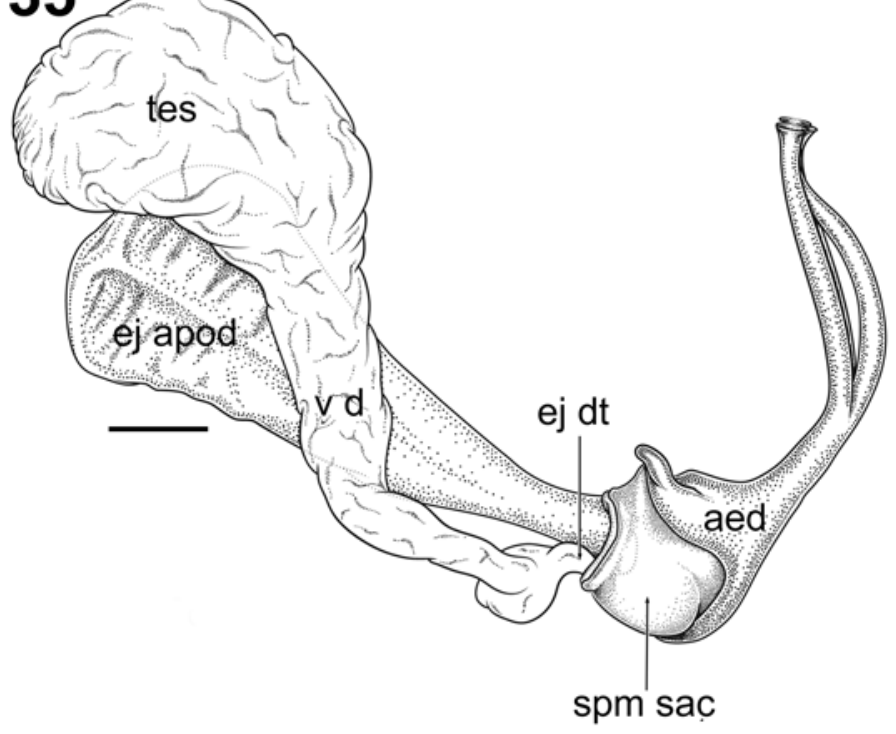

FIGURES 32-35. Araucoderus gloriosus (Alexander) male terminalia and genital tract. 32. Epandrial view. 33. Hypandrial view. 34. Hypandrial view. 35. Lateral view. Abbreviations: aed = aedeagus; ej apod = ejaculatory apodeme; ej dt $=$ ejaculatory duct; epand = epandrium; goncx = gonocoxite; gonst = gonostylus; $\mathrm{pm}$ gbl = paramere lobe at gonocoxite base; $\mathrm{spm}$ sac $=$ sperm sac; tes $=$ testis; $\mathrm{v} \mathrm{d}=$ vas deferens. Scale bars $=0.1 \mathrm{~mm}$ 


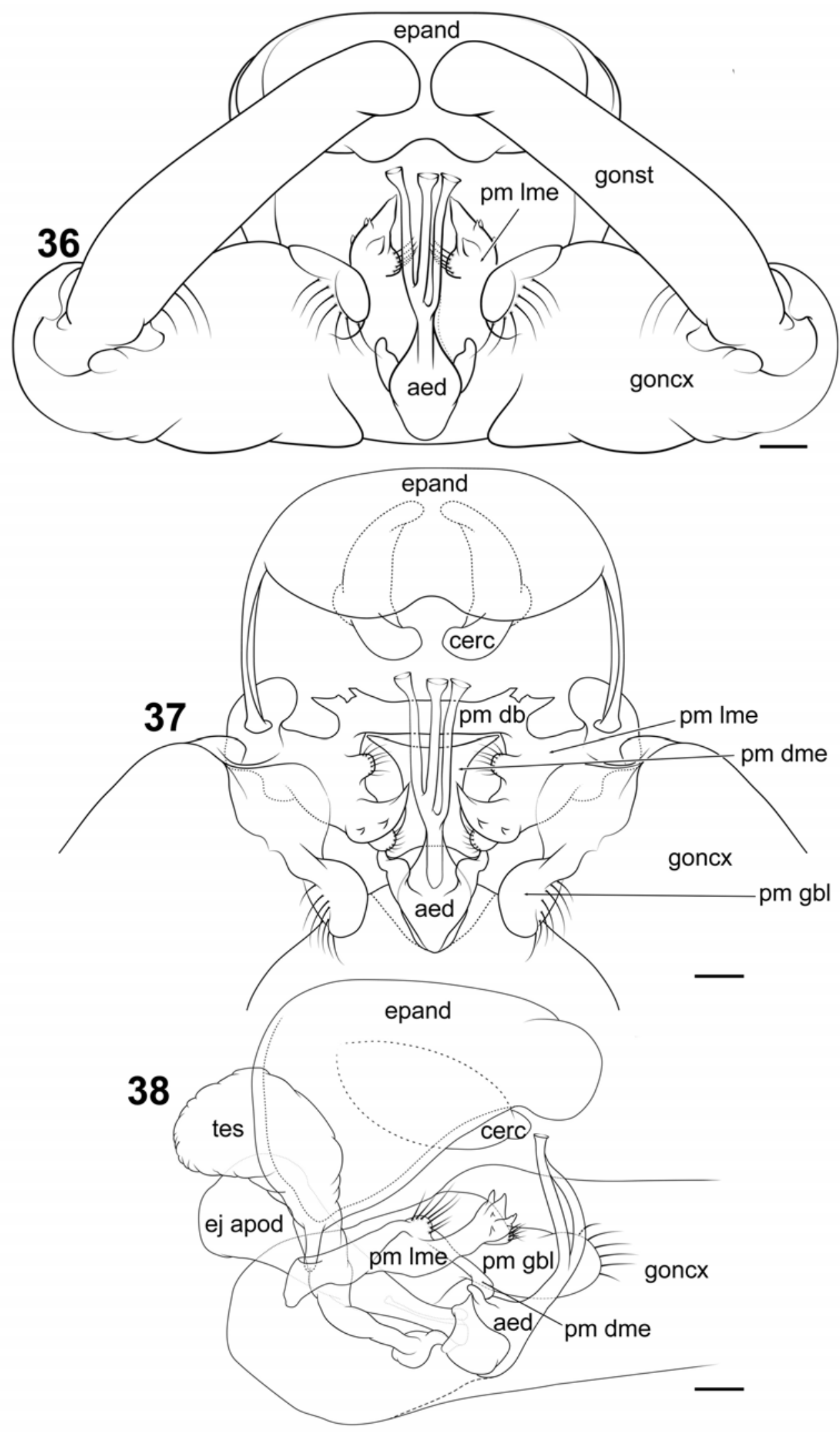

FIGURES 36-38. Araucoderus gloriosus (Alexander) male terminalia. 36. Caudal view. 37. Caudal showing paramere details. 38. Lateral view. Abbreviations; aed = aedeagus; cerc = cercus; ej apod = ejaculatory apodeme; epand = epandrium; goncx = gonocoxite; gonst $=$ gonostylus; $\mathrm{pm} \mathrm{db}=$ dorsal bridge of paramere; $\mathrm{pm}$ dme $=$ dorsomedial element of paramere; $\mathrm{pm}$ gbl $=$ paramere lobe at gonocoxite base; $\mathrm{pm}$ lme = lateromedial element of paramere; tes $=$ testes. Scale bar $=0.10 \mathrm{~mm}$ 

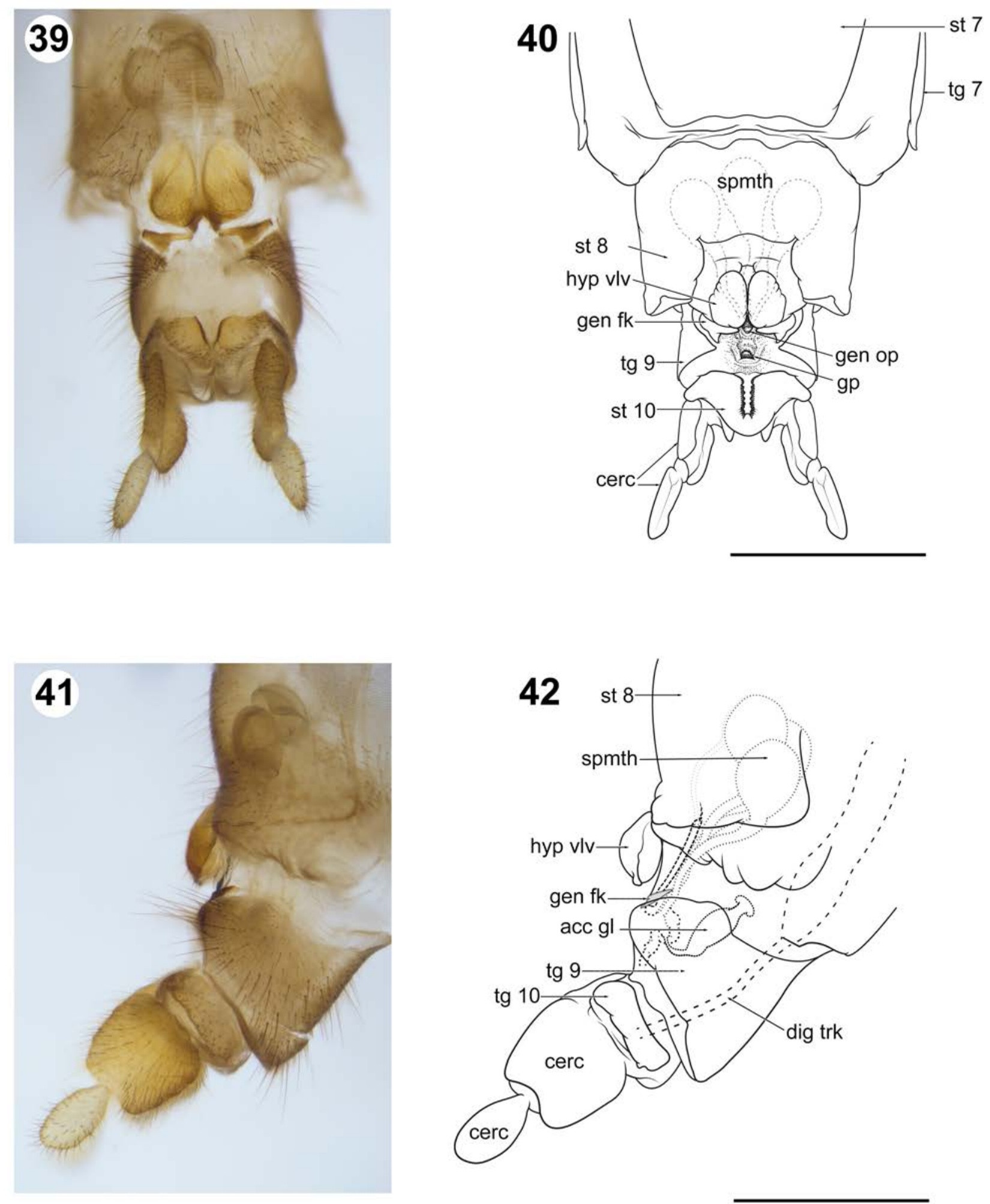

FIGURES 39-42. Araucoderus gloriosus (Alexander) female. 39. Terminalia ventral view. 40. Terminalia ventral view labeled. 41. Terminalia lateral view. 42. Terminalia lateral view labeled. Abbreviations: acc gl = accessory gland; cerc = cercus; dig trk = digestive tract; gen $\mathrm{fk}=$ genital fork; gen op = genital opening; gp = genital papilla; hyp vlv = hypogynial valve; spmth $=$ spermatheca; st 7-st $10=$ sternites \#7-\#10; tg 7-10 = tergites \#7-\#10. Scale bars $=1.00 \mathrm{~mm}$ 


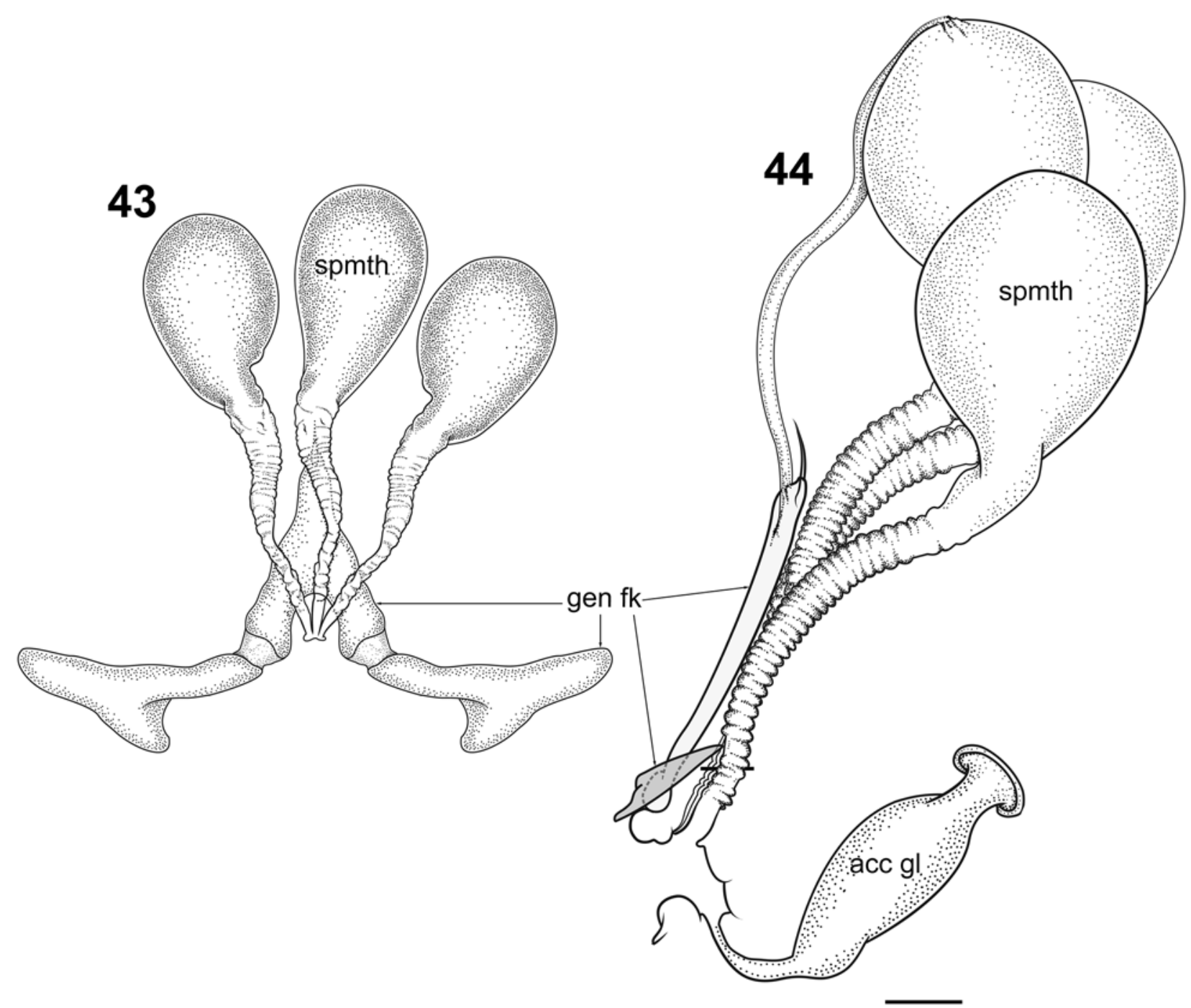

FIGURES 43-44. Araucoderus gloriosus (Alexander) female internal genitalia. 43. Internal genitalia ventral view. 44. Internal genitalia lateral view. Abbreviations: acc $\mathrm{gl}=$ accessory gland; gen $\mathrm{fk}=$ genital fork; spmth $=$ spermatheca. Scale bar $=$ $0.10 \mathrm{~mm}$.

Mating behavior (Fig. 54). On December $13^{\text {th }}$ 2013, a male and female of A. gloriosus were captured and kept alive in a clear container for further observation. Within 30 minutes of capture, the flying male collided several times with the vertically resting female, until coming to rest on the vertical wall of the enclosure side by side. Soon after, the male flexed the abdomen laterally, clasped the female abdomen at mid-length and guided its posterior end toward that of the female using an alternate constricting motion of the gonopods until their terminalia were aligned somewhat perpendicular. The male subsequently turned $180^{\circ}$, displaying a tail to tail copulation position. The copulation event was brief (10-20 seconds), followed by the male dislodging from the female. Neither individual showed further interest toward the other. The same female was observed copulating again several days after the first event. During the second copulatory event, three males were kept in the same enclosure.

During observed copulations, the apical tarsal segment of the foreleg was not used to hold the female, which disagrees with the general mechanism proposed by Hennig (1968). In fact, our observations suggest the female is restrained entirely by the gonopods. The only previous account of tanyderid mating position (Borkent et al. 2008) suggested a generalized orientation in which the mating position after landing was tail to tail while both individuals are standing up. This agrees with our observations except that Araucoderus was unable to stand freely on a horizontal surface.

Our observations of mating in Araucoderus show some similarities with the copulatory behavior of Sycorax silacea Curtis, 1839 (Psychodidae) described by Jung (Jung 1956 p.119) “... The male stands on the side of the 
female, with the body directed in the same direction as her. Suddenly he turns rapidly backwards with open claspers onto the female's abdomen. If the copulation succeeds, both animals stand for about 5 minutes calmly, forming a straight line with their bodies." This observation may be a coincidence, but could be additional evidence supporting a close relationship between Tanyderidae and Psychodidae. Furthermore, Jung's (1956) observations for S. silacea may also characterize the mating behavior of the Neotropical psychodid Aposycorax chilensis Tonnoir, 1929, which exhibits similar behavior and morphology such as short flight pattern, resting in a hanging position and $90^{\circ}$ rotation of the male genitalia (Curler et al. 2015).

Oviposition. Clusters of eggs (Fig. 4-6) were laid on water droplets on the enclosure floor.

Night collections. Previous nocturnal collections of some tanyderids suggest a correlation of flight activity with sunset and evening hours (Judd 2004). Furthermore, black- or incandescent lights can attract some species of Protanyderus Handlirsch (1909), Protoplasa Osten Sacken, 1859 and Mischoderus (Krzemiński \& Judd 1997; Judd 2004; Courtney unpublished observations). During our 2013-2014 expedition, black-light sampling was conducted at different times throughout the night, in areas where immature and adult Araucoderus were present during the afternoon. However, these efforts yielded no adult specimens, suggesting that Araucoderus is not attracted to black lights. On one occasion, with no $A$. gloriosus adults approaching the light and no flight activity observed during sunset hours, a subsequent search of the marginal vegetation revealed nearly two dozen resting $A$. gloriosus adults. Based on our observations, Araucoderus adults may be most active during the morning hours.

Predation. Mid-air predation of adult Araucoderus was observed by a swallow (Passeriformes: Hirundinidae) and a dragonfly (Odonata: Anisoptera). Although no predation of larvae or pupae was observed, we found a dead mature pupa parasitized by over two dozen larval Phoridae.

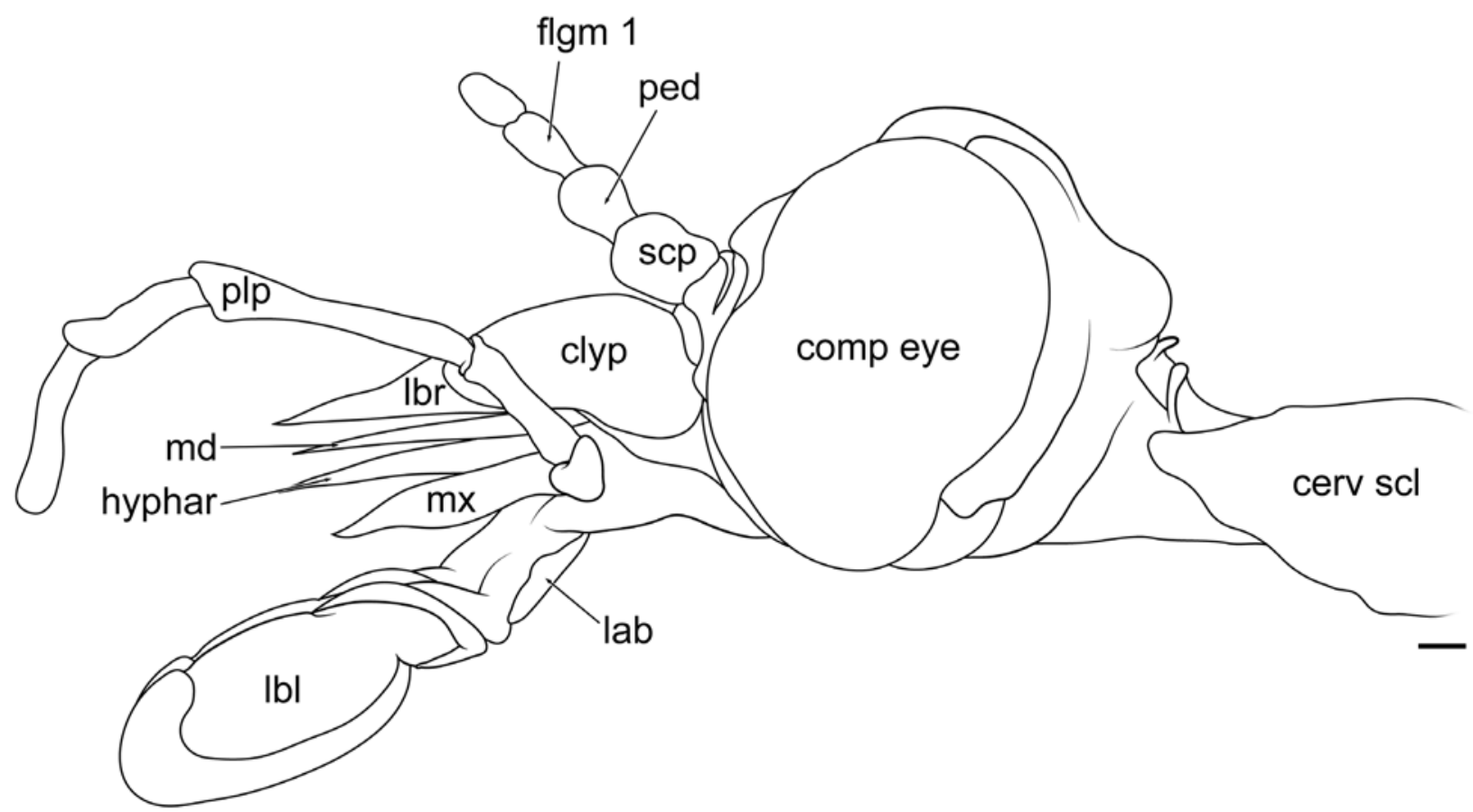

FIGURE 45. Araucoderus gloriosus (Alexander) female. 45. Head capsule lateral view. Abbreviations: cerv scl = cervical sclerite; clyp = clypeus; comp eye = compound eye; flgm $1=$ flagellomere $\# 1$; hyphar $=$ hypopharynx; lab = labium; lbl = labellum; lbr = labrum; $\mathrm{md}=$ mandible; $\mathrm{mx}=$ maxilla; ped $=$ pedicel; plp = palpus; $\mathrm{scp}=$ scape. Scale bar $=0.10 \mathrm{~mm}$

\section{Discussion}

A lack of information about the habitat, behavior, and life history of tanyderids has hampered our understanding of the family and presumably contributed to the scarcity of collection records. In addition to new information on morphology and a redescription of Araucoderus gloriosus, this study has provided valuable new information on the natural history of a tanyderid. It is our hope that the data and observations presented here will ultimately lead to additional collections, information, and insights on the biology of these unusual flies. 

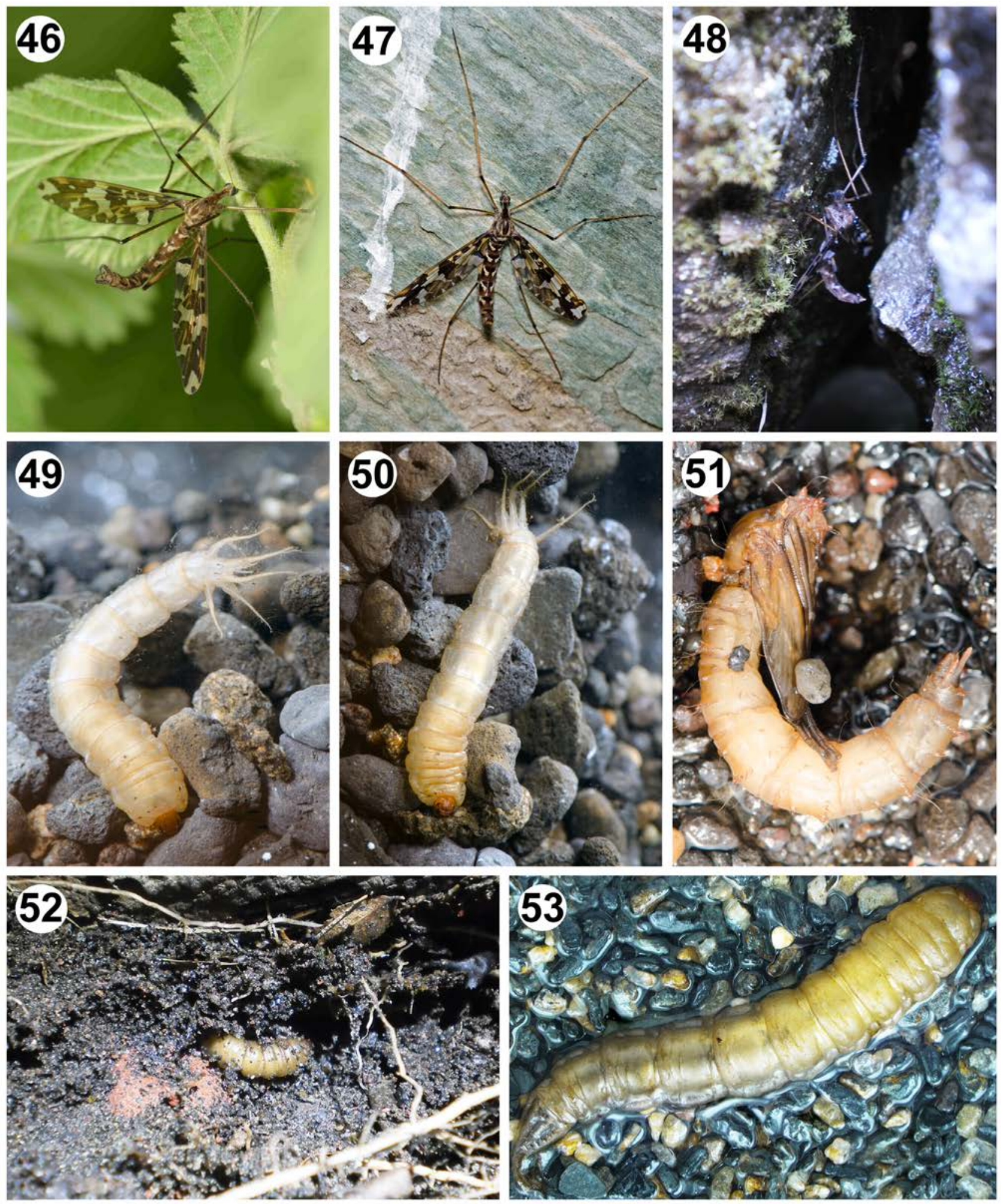

FIGURES 46-53. Araucoderus gloriosus (Alexander) live individuals. 46. Male holding onto underside of riparian vegetation. 47. Female resting vertically on log. 48. Female resting in interstitial space of bridge abutment. $49 \& 50$. Larvae starting to burrow into substrate. 51. Pupa lateral view. 52. Larva burrowed into wet river bank. 53. Larva crawling onto river bank. (Fig. 46 (C) S.A. Marshall; Figs. 47, 49-51 (C) G.W. Courtney). 

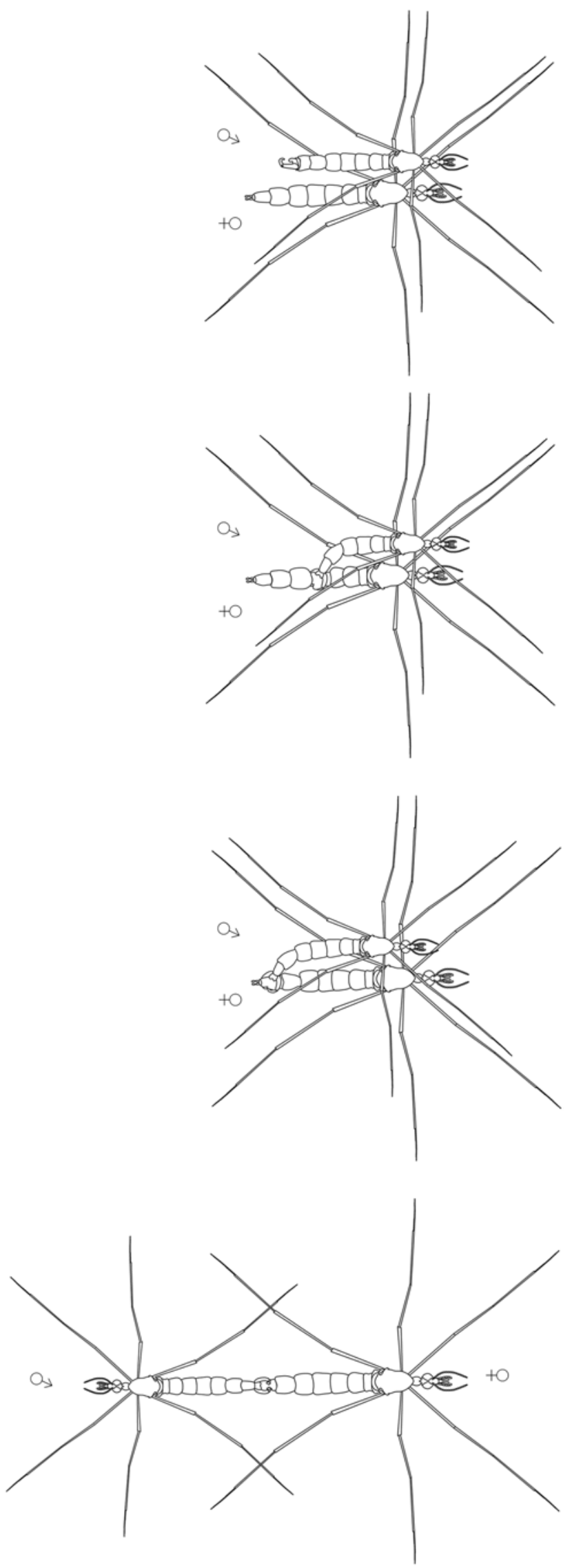

FIGURE 54. Araucoderus gloriosus (Alexander) mating sequence with wings removed. Sexes are distinguished by male and female symbols $(\hat{\jmath}+q)$. 


\section{Acknowledgements}

The authors wish to thank Gregory R. Curler for his valuable comments and information on adult morphology and behavior of Psychodomorpha, as well as his and Art Borkent's careful review of an earlier draft of this paper. Floyd W. Shockley, David G. Furth and Erin Kolski (National Museum of Natural History, Washington D.C., USA) provided some specimens examined during this project. Targe M. Lindsay and Targe M. Lindsay Jr. are thanked for providing valuable literature. Kristina K. Lindsay-Madriz kindly assisted the senior author with fieldwork. Gunnar Kvifte provided valuable insights on the mating position of psychodomorph taxa, especially Sycorax. We thank Steve Marshall for his image of male Araucoderus and Christian R. Gonzalez, Universidad Metropolitana de Ciencias de la Educación, Santiago, and CONAF (Corporación Nacional Forestal) for facilitating our field work in Chile. Special recognition and thanks are extended to Shawna Snyder who illustrated the colored habitus and wing, and to Alexander Mykris for his color illustration of the male head capsule.

This project was supported in part by a National Science Foundation grant (DEB-0933218) to the junior author, and the National Institute of Food and Agriculture, Project No. 6693.

\section{Literature cited}

Alexander, C.P. (1920) A new genus and species of net-winged midge (Blephariceridae) and an undescribed species of Tanyderidae (Diptera). Arkiv för Zoologi, 13, 1-7. http://dx.doi.org/10.5962/bhl.part.20145

Alexander, C.P. (1927) Diptera. Family Tanyderidae. Genera Insectorum, 189, 1-13.

Alexander, C.P. (1929) Diptera of Patagonia and south Chile: based mainly on material in the British Museum (Natural History). Part I. Crane-flies. London: British Museum (Natural History). pp. 228.

Alexander, C.P. (1930) Observations on the dipterous family Tanyderidae. Proceedings of the Linnean Society of New South Wales, 55, 221-230.

Alexander, C.P. (1935) The distribution of Tanyderus pictus Ph. Extracto de la Revista Chilena de Historia Natural, 66, 221259.

Alexander, C.P. (1959) Undescribed species of nematocerous Diptera. Part VII. Bulletin of the Brooklyn Entomological Society, $54,53-59$.

Anthon, H. (1988) Larval morphology of Mischoderus (Insecta, Diptera, Nematocera, Tanyderidae) with notes on tanyderid affinities. Zoologica Scripta, 17, 381-394. http://dx.doi.org/10.1111/j.1463-6409.1988.tb00114.x

Borkent, A., Borkent, C.J. \& Sinclair, B.J. (2008) The male genital tract of Chaoboridae (Diptera: Culicomorpha). The Canadian Entomologist, 140, 621-629. http://dx.doi.org/10.4039/n08-048

Borkent, A. (2012) The pupae of Culicomorpha-morphology and a new phylogenetic tree. Zootaxa, 3396, 1-98.

Borkent, A. \& Sinclair, B.J. (2012) The male genital tract of Axymyiidae and Tanyderidae (Diptera). The Canadian Entomologist, 144, 266-272. http://dx.doi.org/10.4039/tce.2012.26

Colless, D.H. \& McAlpine, D.K. (1970) Diptera (flies). In: The Insects of Australia. Melbourne University Press, pp. 656-740.

Colless, D.H. \& McAlpine, D.K. (1991) Diptera (flies). In: The Insects of Australia. Vol. 2. Cornell University Press, pp. 717786.

Courtney, G.W., Sinclair, B.J. \& Meier, R. (2000). Morphology and terminology of Diptera larvae. In: Papp, L. \& Darvas, B. (Eds.), Contributions to a Manual of Palaearctic Diptera. Vol. 1. Science Herald, Budapest. pp. 85-162.

Courtney, G.W. \& Merritt, R.W. (2008) Aquatic Diptera: Part one: Larvae of aquatic Diptera. In: Merritt, R.W., Cummins, K.W. $\&$ Berg, M.B. (Eds.), An Introduction to the Aquatic insects of North America. $4^{\text {th }}$ Ed. Kendall/Hunt Publishing Co., Dubuque, Iowa. pp. 687-722.

Crampton, G.C. (1930) A comparison of the more important structural details of the larvae of the archaic tanyderid dipteron Protoplasa fitchii with other Holometabola from the standpoint of phylogeny. Brooklyn Entomological Society, 25, 239258.

Cumming, J.M. \& Wood, D.M. (2009) Adult morphology and terminology. In: Brown, B.V., Borkent, A., Cumming, J.M., Wood, D.M., Woodley, N.E. \& Zumbado, M.A. (Eds.), Manual of Central American Diptera: Vol. 1. NRC Research Press, Ottawa, Ontario, Canada. pp. 9-50.

Curler, G.R., Moulton, J.K. \& Madriz, R.I. (2015) Redescription of Aposycorax chilensis (Tonnoir) (Diptera, Psychodidae, Sycoracinae) with the first identification of a blood meal host for the species. Zootaxa, 4048 (1), 114-126. http://dx.doi.org/10.11646/zootaxa.4048.1.7

Exner, K. \& Craig, D.A. (1976) Larvae of Alberta Tanyderidae (Diptera: Nematocera). Quaestiones Entomologicae, 12, 219 237.

Friedemann, K., Schneeberg, K. \& Beutel, R.G. (2014) Fly on the wall-attachment structures in lower Diptera. Systematic Entomology, 39, 460-473. 
http://dx.doi.org/10.1111/syen.12064

Handlirsch, A. (1909) Zur phylogenie und flügelmorphologie der Ptychopteriden (Dipteren). Annalen des Naturhistorischen Museums in Wien, 23, 263-272.

Hennig, W. (1968) Kritische Bemerkungen über den Bau der Flügelwurzel bei den Dipteren und die Frage nach der Monophylie der Nematocera. Stuttgarter Beiträge Naturkunde 193, 1-23.

Hinton, H.E. (1966) The spiracular gill of the fly Eutanyderus (Tanyderidae). Australian Journal of Zoology, 14, $365-369$. http://dx.doi.org/10.1071/ZO9660365

Judd, D.D. (2004) Insecta: Diptera, Tanyderidae. In: Yule, C.M \& Yong, H.S (Eds.), Freshwater Invertebrates of the Malaysian Region. Academy of Sciences Malaysia, pp. 626-633.

Jung, H.F. (1956) Beiträge zur Biologie, Morphologie und Systematik der europäischen Psychodiden (Diptera). Deutsche Entomologische Zeitschrift, 3, 97-257.

Knight, A.W. (1963) Description of the tanyderid larva of Protanyderus margarita Alexander from Colorado. Bulletin of the Brooklyn Entomological Society, 58, 99-102.

Knight, A.W. (1964) Description of the tanyderid pupa of Protanyderus margarita Alexander from Colorado. Entomological News, 75, 237-241.

Kotrba, M. (2000) Morphology and terminology of the female postabdomen. In: Papp, L. \& Darvas, B. (Eds.), Contributions to a Manual of Palaearctic Diptera. Vol. 1. Science Herald, Budapest: pp. 75-84.

Krzemiński, W. \& Judd, D.D. (1997) Family Tanyderidae. In: Papp, L. \& Darvas, B. (Eds.), Contributions to a Manual of Palaearctic Diptera. Vol. 2: Nematocera and Lower Brachycera. Science Herald, Budapest, pp. 281-289.

Lukasevich, E.D. \& Scherbakov, D. (2014) First description of Tanyderidae (Diptera) larvae from South America. Russian Entomological Journal, 23, 121-138.

Lukasevich, E.D. \& Scherbakov, D. (2016) On morphology of Tanyderus pictus (Diptera: Tanyderidae) pupa and adult from Chile. Russian Entomological Journal, 25, 79-95.

Marshall, S.A. (2012) Flies: the natural history and diversity of Diptera. Firefly Books Ltd., Richmond Hill, Ontario, 616 pp.

Osten Sacken, C.R. (1859) New genera and species of North American Tipulidae with short palpi, with attempt at a new classification of the tribe. Proceedings of the Academy of Natural Sciences of Philadelphia, 11, 197-256.

Osten Sacken, C.R. (1880) Die Tanyderina, eine merkwürdige gruppe der Tipuliden. Verhandlungen der KaiserlichKöeniglichen zoologisch-botanischen Gesellschaft in Wien, 29, 517-522.

Philippi, R.A. (1865) Aufzählung der chilenischen Dipteren. Verhandlungen der kaiserlich-königlichen zoologisch-botanischen Gesellschaft in Wien, 15, 595-782.

Podeniene, V. \& Gelhaus, J.K. (2013) Larva of Protanyderus stackelbergi Savchenko, 1971 (Diptera: Ptychopteromorpha, Tanyderidae) from Mongolia. Proceedings of the Academy of Natural Sciences of Philadelphia, 162, 125-132. http://dx.doi.org/10.1635/053.162.0108

Rose, J.H. (1963) Supposed larva of Protanyderus vipio (Osten Sacken) discovered in California (Diptera: Tanyderidae). PanPacific Entomologist, 39, 272-275.

Savchenko, E.N. (1971) On the discovery of tanyderid- flies (Diptera, Tanyderidae) in Mongolia. Entomologicheskoye obozreniye (Entomological Review), 50, 167-170.

Wipfler, B. Courtney, G.W., Craig, D.A. \& Beutel, R.G. (2012) First u-CT-based 3D reconstruction of a dipteran larva-The head morphology of Protanyderus (Tanyderidae) and its phylogenetic implications. Journal of Morphology, 273, 968-980. http://dx.doi.org/10.1002/jmor.20035

Wood, H.G. (1952) The crane-flies of the south-west Cape (Diptera, Tipuloidea). Annals of the South African Museum, 39, 1327. 